\title{
Passive Transparency Compensation for Bilateral Teleoperators with Communication Delays
}

\author{
Erick J. Rodríguez-Seda \\ Department of Weapons and Systems Engineering, United States Naval Academy, Annapolis, MD 21402, USA \\ Correspondence should be addressed to Erick J. Rodríguez-Seda; rodrigue@usna.edu
}

Received 30 September 2014; Revised 12 January 2015; Accepted 28 January 2015

Academic Editor: Shahram Payandeh

Copyright ( 2015 Erick J. Rodríguez-Seda. This is an open access article distributed under the Creative Commons Attribution License, which permits unrestricted use, distribution, and reproduction in any medium, provided the original work is properly cited.

\begin{abstract}
One of the main challenges in the realization of time-delayed bilateral teleoperators is the stable adaptation of transparency when the remote environmental dynamics are time-varying. In this paper, we propose a bilateral control strategy that passively adjusts the transparency of the system when the slave robot transitions between two different environments. The proposed controller exploits the effect that the wave impedance (a design parameter of the passivity-based scattering transformation) has on transparency without comprising closed-loop stability, regardless of time-varying communication delays. To properly adjust transparency, the control scheme smoothly switches the wave impedance parameter between a low value, ideal for free motion, and a sufficiently large value, suited for hard-contact tasks. We show that, by adopting this strategy, the transmitted impedance to the operator approximates more closely the environmental impedance value. Furthermore, we theoretically prove master-slave position coordination and force tracking under different scenarios. Simulation results illustrate the effectiveness of the proposed control strategy.
\end{abstract}

\section{Introduction}

A bilateral teleoperator is a dual robotic system that enables a human operator to manipulate, sense, and physically interact with a remote environment. It consists of a local, human-controlled master robot energetically coupled via a communication channel with a remote slave robot. Ideally, the coupling between master and slave robots should be transparent to the operator, meaning that the operator should feel as if he or she is directly active in the remote location [1]. This requires the system to faithfully transmit the environmental impedance to the human operator [1] or, alternatively, to equate the positions and forces at the master and slave sides [2]. Unfortunately, a perfectly transparent bilateral teleoperation system cannot be realized without compromising stability due to the presence of time delays in the communication channel [3].

Challenges to the realization of stable and transparent teleoperators have been well documented in the literature $[1,3-6]$. Particularly, one of the main challenges faced by bilateral teleoperators is how to adjust transparency when the mechanical and dynamical properties of the remote environment change over time. For example, consider the teleoperation system of Figure 1. The remote environment transitions from a section of zero impedance (where the slave robot is free to move) to a restricted, hard-contact section of high impedance (i.e., the wall). Typically, the transmitted impedance to the human operator would be tuned according to one of the two environmental impedance values or a trade-off between both. In the case where large impedance is transmitted to the operator, the teleoperation system will feel sluggish or misleadingly heavy during free motion [7]. If, on the other hand, low impedance value is chosen, substantial discrepancies between the position of the master and slave robots will appear when interacting with the wall (see Figure 1) [8]. In either case, the human operator will lose the ability to accurately feel and identify the mechanical and dynamical properties of the remote environment. Therefore, it is important to adjust the transparency of the teleoperation system according to the current remote environmental dynamics [7].

\section{Background and Contributions}

2.1. Related Work. Prompted by the need for transparent yet stable time-delayed teleoperators, several control frameworks 

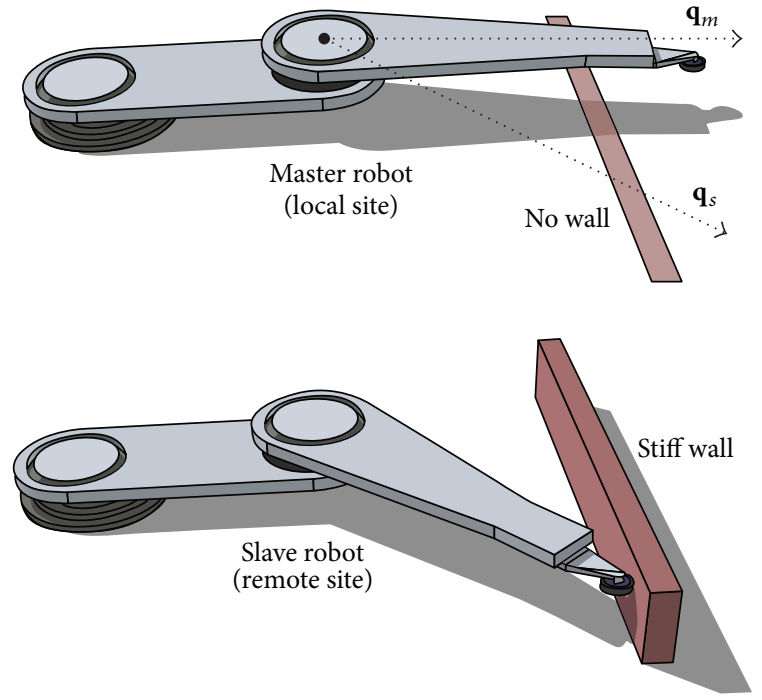

FIgURE 1: Example of a master-slave teleoperation system. If the transmitted impedance to the human operator is not sufficiently large, the operator will feel a softer remote environment and may still continue the motion of the master robot despite the slave robot's position being locked by the wall.

have been proposed (refer to $[9,10]$ for a review of control schemes and a comparison of different methods). Arguably, two of the most dominant control approaches are passivity-based methods, which include the wave-based scattering transformation [11, 12], and traditional proportionalderivative (PD) controllers [13-15]. Yet, most of these control frameworks fail to transmit faithful impedance to the operator when the environmental dynamics are unknown or timevarying. For instance, in passive wave-based architectures [12], transparency depends highly on a control parameter, namely, the wave impedance. For free motion, the ideal wave impedance should be infinitesimal, such that the increase of inertia induced by the delay is barely perceived by the operator. In contrast, for stiff or hard-contact environments, the desired wave impedance should be infinitely large, such that a stiff environment is felt by the operator [16]. Compromising the value of the wave impedance to best satisfy both scenarios tends to lead to a teleoperation system that feels sluggish in free motion with substantial position errors when interacting with stiff environments. These position errors, also referenced as position drifts [8], cause the operator to erroneously interpret the actual position of the slave (e.g., see Figure 1). A similar effect is experienced when tuning traditional proportional-derivative (PD) architectures where control gains are limited by stability constrains and, consequently, position errors arise while in contact motion $[6,8]$.

Time-varying compensation of position errors during contact tasks, which aimed to improve static transparency, has been addressed previously in [17] via a wave-based scheme that introduces the notion of a variable rest length. The role of the variable rest length is to modify the desired target position according to the position drift and applied forces so that the master-slave position error converges to zero. A similar approach based on the variable rest length is presented in [18], where an energy tank replaces the dissipative element in the wave scattering transformation for impedance matching so that the energy is stored rather than dissipated. The stored energy is then used to adequately change the variable rest length without relying on the operator's energy as done in [17]. In both of the above methods, the communication delay must be known and constant in order to perform the position compensation.

In [19], a delay-independent, wave-based control framework was proposed where the wave impedance passively switches between an arbitrary small value, ideal for free motion, and an arbitrary large value, suited for stiff environments. The idea of modifying the wave impedance according to the remote environment was previously explored in [20]. However, the switching policy of [20] requires the mechanical and control systems to dissipate a certain amount of energy (proportional to the difference in impedance values) to execute a stable transition. The work of [19] has been preliminarily extended to the case of time-varying communication delays [21].

Other recent compensation methods not based on wavebased scattering transformation include the work in [22-24]. In [22], a two-layer approach is proposed where the first layer is designed to improve the transparency of the system based on the knowledge of the task and environment, while the second layer guarantees that passivity is preserved. In [23], a switching two-channel control architecture is developed for linear systems with constant delays. The overall control architecture switches between two controllers that are individually optimized for free and hard-contact motion. Finally, in [24], a switching control strategy for linear teleoperators is presented; however, it does not take into account the presence of communication delays.

2.2. Contributions. The main contribution of the paper is the proof of the system's closed-loop stability regardless of the variability of the wave impedance and communication delays as well as the state convergence analysis for several special cases. The paper formally extends and unifies the time-varying wave impedance control architecture of $[19,21]$ to bilateral teleoperators with time-varying communication delays. We start by introducing the passivity-based scattering transformation with position information [25] and analyze how the wave impedance and the delay affect transparency for two different remote environments: one with a zero impedance and another with high impedance. Among our findings, we illustrate how can we exploit the wave impedance to improve the transparency of the teleoperation system. Using this knowledge, we then propose an update strategy that smoothly adjusts the wave impedance value according to the current environmental dynamics without compromising the stability of the closed-loop system. In contrast to [19], the transitions between wave impedance values can be executed arbitrarily fast. Finally, we remove the need of transmitting remote contact information to the master robot by updating the wave impedance at the slave side rather than at the 
master side and prove stability and position coordination under different scenarios. Simulation results with a nonlinear teleoperator validate the proposed control architecture.

2.3. Notation. As standard notation, let $\Re^{n}$ be the $n$ dimensional Euclidean space and let $\mathscr{C}=\mathscr{C}\left([-r, 0], \mathfrak{R}^{n}\right)$ be the space of continuous functions taking the interval $[-r, 0]$ into $\mathfrak{R}^{n}$ for $r \geq 0$. If $\mathbf{x}$ is a continuous function defined in the interval $[-r, \alpha)$, where $\alpha>0$, then $\mathbf{x}_{t}$ will denote an element of $\mathscr{C}$ for each $t$ in $[0, \alpha)$ defined by $\mathbf{x}_{t}:=\mathbf{x}(t+\theta)$, $\theta \in[-r, 0]$ [26]. Similarly, the 2-norm of a vector $\mathbf{x}=$ $\left[x_{1}, \ldots, x_{n}\right]^{T}$ will be defined as $\|\mathbf{x}\|:=\sqrt{x_{i}^{2}+\cdots+x_{n}^{2}}$ and the minimum and maximum eigenvalues of a square matrix $A$ as $\underline{\sigma}(A)$ and $\bar{\sigma}(A)$, respectively. Finally, we say that a $n$ dimensional piecewise continuous function $\mathbf{x}:[0, \infty) \rightarrow$ $\mathfrak{R}^{n}$ belongs to $\mathscr{L}_{2}$ if $\|\mathbf{x}(t)\|_{\mathscr{L}_{2}}=\sqrt{\int_{0}^{\infty}\|\mathbf{x}(\theta)\|^{2} d \theta}<\infty$ and to $\mathscr{L}_{\infty}$ if $\|\mathbf{x}(t)\|_{\mathscr{L}_{\infty}}=\sup _{t \geq 0}\|\mathbf{x}(t)\|<\infty$. For simplicity, we will omit time dependence of signals except when considered necessary.

\section{Problem Formulation}

3.1. Modeling the Teleoperators. We address the task of remotely controlling an $n$-degree-of-freedom (DOF) slave robot coupled bilaterally to an $n$-DOF master robot through a time-delayed communication channel. The master and slave robots have nonlinear Euler-Lagrangian dynamics given by

$$
\begin{gathered}
M_{m}\left(\mathbf{q}_{m}\right) \ddot{\mathbf{q}}_{m}+C_{m}\left(\mathbf{q}_{m}, \dot{\mathbf{q}}_{m}\right) \dot{\mathbf{q}}_{m}+\mathbf{g}_{m}\left(\mathbf{q}_{m}\right)=\mathbf{f}_{m}+\overline{\boldsymbol{\tau}}_{m}, \\
M_{s}\left(\mathbf{q}_{s}\right) \ddot{\mathbf{q}}_{s}+C_{s}\left(\mathbf{q}_{s}, \dot{\mathbf{q}}_{s}\right) \dot{\mathbf{q}}_{s}+\mathbf{g}_{s}\left(\mathbf{q}_{s}\right)=\mathbf{f}_{s}+\overline{\boldsymbol{\tau}}_{s},
\end{gathered}
$$

where $\mathbf{q}_{i}=\mathbf{q}_{i}(t) \in \mathfrak{R}^{n}$ are the generalized coordinates, $M_{i}\left(\mathbf{q}_{i}\right) \in \Re^{n \times n}$ are the bounded, positive definite inertia matrices, $C_{i}\left(\mathbf{q}_{i}, \dot{\mathbf{q}}_{i}\right) \in \mathfrak{R}^{n \times n}$ are the centrifugal and Coriolis matrices, $\mathbf{g}_{i}\left(\mathbf{q}_{i}\right)$ are the gravitational forces, $\mathbf{f}_{i}=\mathbf{f}_{i}(t) \in \mathfrak{R}^{n}$ are the human and environmental forces, and $\overline{\boldsymbol{\tau}}_{i}=\overline{\boldsymbol{\tau}}_{i}(t) \in \mathfrak{R}^{n}$ are the control inputs for the master $(i=m)$ and slave robots $(i=s)$. Due to its Euler-Lagrangian dynamic structure, the $j k$ th element of $C_{i}\left(\mathbf{q}_{i}, \dot{\mathbf{q}}_{i}\right)$ is given by

$$
C_{i}^{j k}\left(\mathbf{q}_{i}, \dot{\mathbf{q}}_{i}\right)=\sum_{l=1}^{n} \frac{1}{2}\left[\frac{\partial M_{i}^{j k}}{\partial q_{i}^{l}}+\frac{\partial M_{i}^{j l}}{\partial q_{i}^{k}}-\frac{\partial M_{i}^{k l}}{\partial q_{i}^{l}}\right] \dot{q}_{i}^{l},
$$

where $q_{i}^{l}$ stands for the $l$ th element of $\mathbf{q}_{i}$. Therefore, (1) satisfies the well known passivity property

$$
\dot{M}_{i}\left(\mathbf{q}_{i}\right)=C_{i}\left(\mathbf{q}_{i}, \dot{\mathbf{q}}_{i}\right)+C_{i}^{T}\left(\mathbf{q}_{i}, \dot{\mathbf{q}}_{i}\right) .
$$

In addition, we assume that $M_{i}\left(\mathbf{q}_{i}\right)$ is upper and lower bounded, which in turns implies that $\left\|C_{i}\left(\mathbf{q}_{i}, \dot{\mathbf{q}}_{i}\right)\right\| \leq \kappa_{C_{i}}\left\|\dot{\mathbf{q}}_{i}\right\|$ for some $\kappa_{C_{i}} \geq 0$.

3.2. Control Objectives. Our control goal is to design the inputs $\overline{\boldsymbol{\tau}}_{i}$ such that stability and transparency of the closeloop system in (1) are achieved. Explicitly, we would like $\overline{\boldsymbol{\tau}}_{i}$ to enforce position coordination for finite delays, that is,

$$
\mathbf{q}_{m}(t)-\mathbf{q}_{s}(t) \longrightarrow 0
$$

and static force reflection, that is,

$$
\mathbf{f}_{m}(t) \longrightarrow-\mathbf{f}_{s}(t)
$$

as $\dot{\mathbf{q}}_{i} \rightarrow 0$, independently of the structure of the remote environment. Furthermore, we would like the operator to perceive low and high impedances when interacting with free and rigid environments, respectively.

3.3. Assumptions. In the following analysis we make the assumption that delays on the transmission lines from master to slave, $T_{m}(t)$, and from slave to master, $T_{s}(t)$, are finite (i.e., $\exists T>0$ such that $\left.T_{m}(t)+T_{s}(t) \leq T\right)$ but not necessarily equal and/or constant. Furthermore, we assume that the slave robot is equipped with force/torque sensors (or contact/proximity sensors) that can measure contact information.

\section{Bilateral Control Framework}

In this section we will develop the bilateral control framework to guarantee stability and transparency. First, we will address the problem of stability through the passivity formalism since, in general, passivity is a sufficient condition for the stability of serially connected systems. Then, we will proceed to guarantee transparency-based objectives.

Definition 1 (see [27]). A system with input $\mathbf{x}$ and output $\mathbf{y}$ is said to be passive if

$$
\int_{0}^{t} \mathbf{x}^{T} \mathbf{y} d \theta \geq-\kappa^{2}+\nu^{2} \int_{0}^{t} \mathbf{x}^{T} \mathbf{x} d \theta+\rho^{2} \int_{0}^{t} \mathbf{y}^{T} \mathbf{y} d \theta
$$

for some $\kappa, \nu, \rho \in \mathfrak{R}$. Moreover, it is said to be lossless if equality persists and $\nu=\rho=0$, input strictly passive if $v \neq 0$, and output strictly passive if $\rho \neq 0$.

In order to passivize and hence stabilize the teleoperator, we propose the design of the control inputs as

$$
\overline{\boldsymbol{\tau}}_{i}=\boldsymbol{\tau}_{i}-M_{i}\left(\mathbf{q}_{i}\right) \Lambda \dot{\mathbf{q}}_{i}-C_{i}\left(\mathbf{q}_{i}, \dot{\mathbf{q}}_{i}\right) \Lambda \mathbf{q}_{i}+\mathbf{g}_{i}\left(\mathbf{q}_{i}\right)-K_{i} \dot{\mathbf{q}}_{i},
$$

where $\Lambda \in \mathfrak{R}^{n \times n}$ and $K_{i} \in \mathfrak{R}^{n \times n}$ are, without loss of generality, diagonal positive definite constant matrices and $\boldsymbol{\tau}_{i}=\boldsymbol{\tau}_{i}(t) \epsilon$ $\mathfrak{R}^{n}$ are the coordination control inputs to be designed. Then, the dynamic equations of the system in (1) reduce to

$$
M_{i}\left(\mathbf{q}_{i}\right) \dot{\mathbf{r}}_{i}+C_{i}\left(\mathbf{q}_{i}, \dot{\mathbf{q}}_{i}\right) \mathbf{r}_{i}=\mathbf{f}_{i}+\boldsymbol{\tau}_{i}-K_{i} \dot{\mathbf{q}}_{i},
$$

where $\mathbf{r}_{i}(t)=\dot{\mathbf{q}}_{i}(t)+\Lambda \mathbf{q}_{i}(t)$.

Remark 2. Note that the control law in (7) assumes complete knowledge of the dynamics of the master and slave robot. In $[25,28]$, a passivity-based adaptive law is suggested for the case where the parameters are unknown. Such approach can be easily extended to our proposed control framework without altering the main results presented herein.

We are now left to design the control inputs $\boldsymbol{\tau}_{i}$ such that the communication channel is passivized independently of the delay and that position convergence and force tracking of 
the teleoperation system are guaranteed. With this in mind, we propose the use of the scattering transformation and wave variables $\mathbf{u}_{i}$ and $\mathbf{v}_{i}[11,12]$. For the slave side, the outputs of the scattering transformation are computed as

$$
\begin{aligned}
& \mathbf{v}_{s}(t)=\left(2 B_{s}(t)\right)^{-1 / 2}\left(B_{s}(t) \mathbf{r}_{s d}(t)-\boldsymbol{\tau}_{s}(t)\right), \\
& \mathbf{r}_{s d}(t)=\left(2 B_{s}^{-1}(t)\right)^{1 / 2} \mathbf{u}_{s}(t)-B_{s}^{-1}(t) \boldsymbol{\tau}_{s}(t),
\end{aligned}
$$

where $B_{s}(t) \in \mathfrak{R}^{n \times n}$, the wave impedance, is a bounded, diagonal, time-varying, positive definite matrix that will be designed under transparency concerns; and $\mathbf{u}_{s}(t)=$ $\gamma_{s}(t) \mathbf{u}_{m}\left(t-T_{m}(t)\right)$ is the incoming wave variable from the master's scattering transformation scaled by some positive semidefinite scalar function $\gamma_{s}$. Then, the coordination control input can be computed as

$$
\boldsymbol{\tau}_{s}(t)=B_{s}(t)\left(\mathbf{r}_{s d}(t)-\mathbf{r}_{s}(t)\right) .
$$

Likewise, for the master side, the outputs of the scattering transformation are computed as

$$
\begin{gathered}
\mathbf{u}_{m}(t)=\left(2 B_{m}(t)\right)^{-1 / 2}\left(B_{m}(t) \mathbf{r}_{m d}(t)-\boldsymbol{\tau}_{m}(t)\right), \\
\mathbf{r}_{m d}(t)=\left(2 B_{m}^{-1}(t)\right)^{1 / 2} \mathbf{v}_{m}(t)-B_{m}^{-1}(t) \boldsymbol{\tau}_{m}(t),
\end{gathered}
$$

where $B_{m}(t)=B_{s}\left(t-T_{s}(t)\right)$ and $\mathbf{v}_{m}(t)=\gamma_{m}(t) \mathbf{v}_{s}\left(t-T_{s}(t)\right)$ for some positive semidefinite function $\gamma_{m}$. Analogous to the slave case,

$$
\boldsymbol{\tau}_{m}(t)=B_{m}(t)\left(\mathbf{r}_{m d}(t)-\mathbf{r}_{m}(t)\right) .
$$

The reader can easily verify that using the scattering transformation and the coordination control inputs (11) and (14), (10) and (13) reduce to

$$
\begin{gathered}
2 \mathbf{r}_{s d}(t)=\left(\gamma_{s}(t) \Gamma(t) \mathbf{r}_{m}\left(t-T_{m}(t)\right)+\mathbf{r}_{s}(t)\right), \\
2 \mathbf{r}_{m d}(t)=\left(\gamma_{m}(t) \mathbf{r}_{s}\left(t-T_{s}(t)\right)+\mathbf{r}_{m}(t)\right),
\end{gathered}
$$

where $\Gamma(t)=B_{s}\left(t-T_{m}(t)-T_{s}\left(t-T_{m}(t)\right)\right)^{1 / 2} B_{s}(t)^{-1 / 2}$.

Now, let us show that the scattering transformation with the appropriate choice of $\gamma_{i}$ guarantees the passivity of the communication channel independently of delays and variance of the wave impedance. Manipulating (9) to (14), we obtain that the power equation for the communication channel is given by

$$
-\left(\boldsymbol{\tau}_{m}^{T} \mathbf{r}_{m d}+\boldsymbol{\tau}_{s}^{T} \mathbf{r}_{s d}\right)=\frac{1}{2}\left(\mathbf{u}_{m}^{2}-\mathbf{v}_{m}^{2}+\mathbf{v}_{s}^{2}-\mathbf{u}_{s}^{2}\right),
$$

where the negative sign at the left side of the equation is owed to the power inflow. Integrating (17) with respect to time yields the total energy on the communication channel; that is,

$$
\begin{aligned}
-\int_{0}^{t}\left(\boldsymbol{\tau}_{m}^{T}(\theta) \mathbf{r}_{m d}(\theta)+\boldsymbol{\tau}_{s}^{T}(\theta) \mathbf{r}_{s d}(\theta)\right) d \theta \\
=\frac{1}{2} \int_{0}^{t}\left(\mathbf{u}_{m}^{2}(\theta)-\gamma_{s}(\theta) \mathbf{u}_{m}^{2}\left(\theta-T_{m}(\theta)\right)\right) d \theta \\
\quad+\frac{1}{2} \int_{0}^{t}\left(\mathbf{v}_{s}^{2}(\theta)-\gamma_{m}(\theta) \mathbf{v}_{s}^{2}\left(\theta-T_{s}(\theta)\right)\right) d \theta .
\end{aligned}
$$

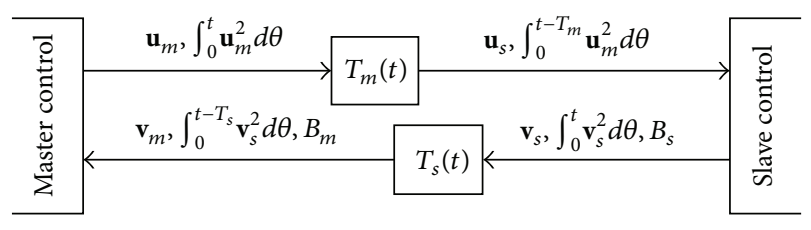

FIGURE 2: Flow of information between master and slave robots.

Note that the energy is independent of the value of the wave impedance. It, however, depends on the characteristics of the delay and the choice of $\gamma_{i}$.

4.1. Time Constant Delays. Let us consider first the case of constant communication delays, that is, $T_{m}(t)=T_{m}$ and $T_{s}(t)=T_{s}$. Define $\gamma_{m}(t)=\gamma_{s}(t)=1$, for all $t \geq 0$. Returning to (18) we obtain that

$$
\begin{aligned}
& -\int_{0}^{t}\left(\boldsymbol{\tau}_{m}^{T}(\theta) \mathbf{r}_{m d}(\theta)+\boldsymbol{\tau}_{s}^{T}(\theta) \mathbf{r}_{s d}(\theta)\right) d \theta \\
& \quad=\frac{1}{2} \int_{t-T_{m}}^{t} \mathbf{u}_{m}^{2}(\theta) d \theta+\frac{1}{2} \int_{t-T_{s}}^{t} \mathbf{v}_{s}^{2}(\theta) d \theta \geq 0
\end{aligned}
$$

which yields that the communication channel is passive (for $\kappa=0$ ) independently of arbitrary large constant delays.

4.2. Time-Varying Delays. We have demonstrated that the use of the conventional wave scattering transformation (for $\left.\gamma_{i}(t)=1\right)$ guarantees the passivity of the communication channel independently of the value of the wave impedance when the delays are constant. The problem arises due to the compression/expansion of the transmitted wave variables when the delays are time-varying. To guarantee that the communication is passive, we must guarantee that the output energy is not greater than the energy coming into the communication channel. A sufficient condition for passivity is then given by the following two inequalities:

$$
\begin{gathered}
\int_{0}^{t} \mathbf{u}_{m}^{2}(\theta) d \theta \geq \int_{0}^{t} \gamma_{s}^{2}(\theta) \mathbf{u}_{m}^{2}\left(\theta-T_{m}(\theta)\right) d \theta \\
\int_{0}^{t} \mathbf{v}_{s}^{2}(\theta) d \theta \geq \int_{0}^{t} \gamma_{m}^{2}(\theta) \mathbf{v}_{s}^{2}\left(\theta-T_{s}(\theta)\right) d \theta
\end{gathered}
$$

Therefore, we will design $\gamma_{s}$ and $\gamma_{m}$ such that (20) are satisfied. To this end, we propose to transmit the total energy of the incoming wave variables along with the wave variables (see Figure 2 for a pictorial representation) and to design two energy filters. The equations governing $\gamma_{s}$ and $\gamma_{m}$ are given by

$$
\gamma_{i}= \begin{cases}1, & \text { if } E_{i} \geq \beta_{i} \\ \frac{2 \beta_{i}^{2} E_{i}^{2}}{E_{i}^{4}+\beta_{i}^{4}}, & \text { otherwise }\end{cases}
$$




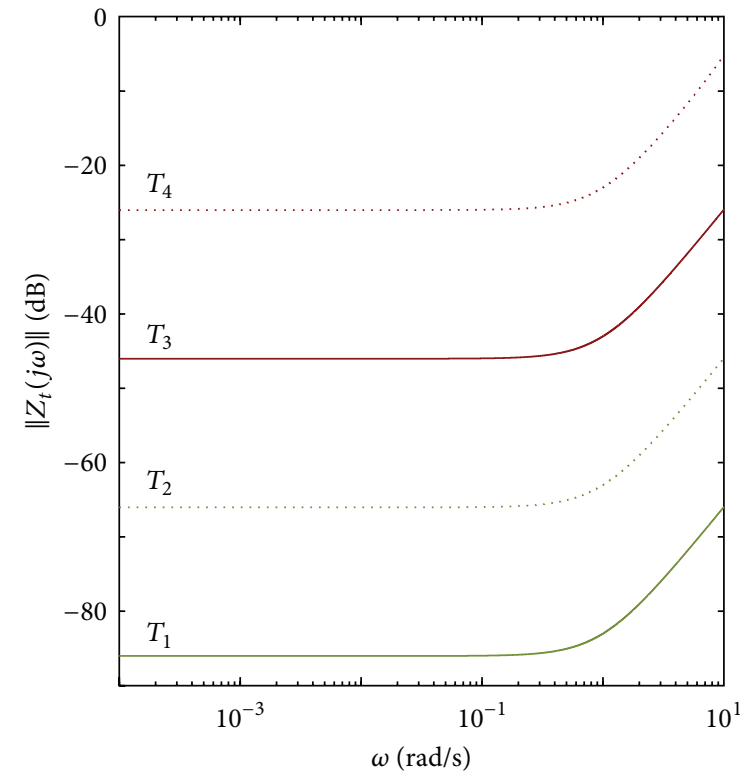

(a) $\left\|Z_{t}(j \omega)\right\|$ as a function of $T$

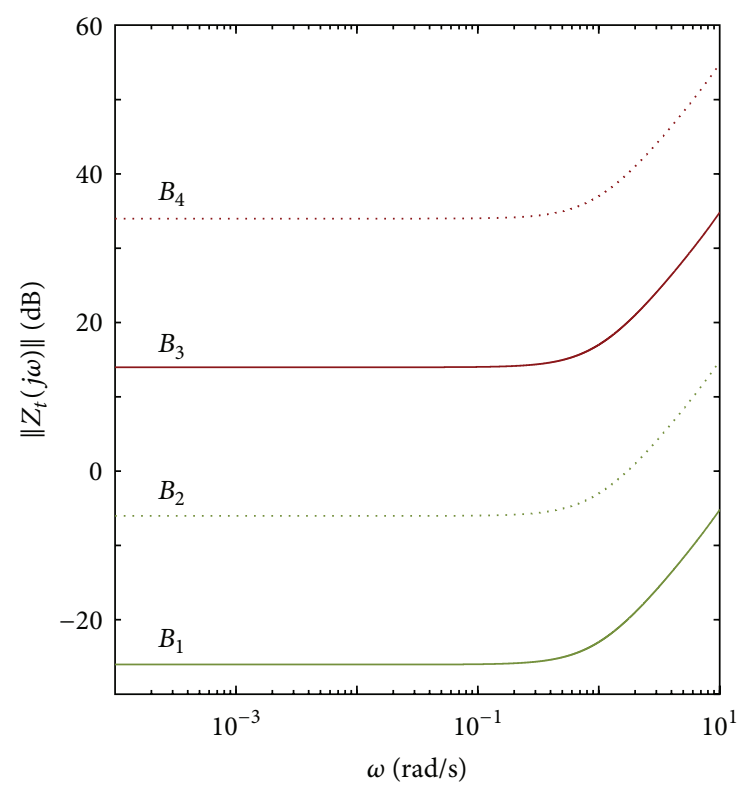

(b) $\left\|Z_{t}(j \omega)\right\|$ as a function of $B$

FIGURE 3: Bode magnitude plot of transmitted impedance $Z_{t}(s)$ for the free motion case. (a) $\left\|Z_{t}(j \omega)\right\|$ plotted for different delays when $B=1[\mathrm{~N} \cdot \mathrm{m} / \mathrm{s}]$ is fixed. (b) $\left\|Z_{t}(j \omega)\right\|$ plotted for different wave impedances when $T=0.1[\mathrm{~s}]$ is fixed.

where $\beta_{i}$ are positive constants for $i \in\{m, s\}$ and

$$
\begin{aligned}
& E_{m}=\int_{0}^{t-T_{s}(t)} \mathbf{v}_{s}^{2}(\theta) d \theta-\int_{0}^{t} \mathbf{v}_{m}^{2}(\theta) d \theta, \\
& E_{s}=\int_{0}^{t-T_{m}(t)} \mathbf{u}_{m}^{2}(\theta) d \theta-\int_{0}^{t} \mathbf{u}_{s}^{2}(\theta) d \theta
\end{aligned}
$$

are the energy stored (also called energy reservoirs) in the communication channels. Note that the energy is never negative, since $E_{i} \rightarrow 0 \Rightarrow \gamma_{i} \rightarrow 0$, and thus, the outgoing wave variable also converges to zero. Therefore, (20) are satisfied for all $t$ and the communication channel is passive.

Up to now, we have designed the control inputs $\overline{\boldsymbol{\tau}}_{i}$ based on passivity concerns. We are yet to tune the control law such that transparency is achieved for both free and restricted environments. To this end, we will exploit the effect of the wave impedance on position convergence and force reflection.

\section{Tuning of the Wave Impedance}

5.1. Effect of the Wave Impedance on Transparency. According to [1], transparency is achieved when the transmitted impedance to the operator $Z_{t}$ is equal to the environmental impedance $Z_{e}$. This implies that the human operator should feel very little resistance or impedance when the slave robot is in free motion (i.e., $Z_{e}=0$ ) and should feel a higher stiffness or impedance when the motion of the slave is restricted (i.e., $Z_{e}$ is large). In this section, we will show how the wave impedance and the delay affect $Z_{t}$ in comparison to $Z_{e}$. We evaluate two different scenarios: one for which the slave robot can move freely in space and another in which the slave robot is in contact with a stiff surface. For simplicity, we reduce the dynamics of the master and slave robot to 1-DOF linear systems and let $\Lambda=1[1 / \mathrm{s}]$. We assume that the wave impedance $B_{i}=B$ and the roundtrip delay $T=T_{m}+T_{s}$ are constant and that $\mathbf{f}_{m} \approx \boldsymbol{\tau}_{m}$ and $\mathbf{r}_{s} \approx \mathbf{r}_{s d}$. The later implies that the dynamics of the robots are negligible, as in [29].

Now, let us consider the scattering transformation equations given in (9) to (14). Using the Laplace transform we can compute the transmitted impedance to the operator as

$$
Z_{t}(s)=\frac{\mathscr{L}\left\{\mathbf{f}_{m}(t)\right\}}{\mathscr{L}\left\{\dot{\mathbf{q}}_{m}(t)\right\}}=B \frac{s+1}{s} \frac{1+R(s) e^{-s T}}{1-R(s) e^{-s T}},
$$

where $s=j \omega$ denotes the Laplace variable and

$$
R(s)=\frac{Z_{e}(s)-B}{Z_{e}(s)+B}, \quad Z_{e}(s)=\frac{\mathscr{L}\left\{\mathbf{f}_{s}(t)\right\}}{\mathscr{L}\left\{\dot{\mathbf{q}}_{s}(t)\right\}} .
$$

To evaluate the effect of the delay and the wave impedance, we will plot the magnitude of $Z_{t}$ for two different $Z_{e}$. For the handling of the exponential in (23), we will use a Padé approximation of order $N_{P}=\left\lceil 6 \pi f_{P} T\right\rceil$, where $f_{P}$ is the maximum frequency for which the Padé approximation of order $N_{p}$ yields an accurate representation of the delay [29]. For simplicity, we choose a low frequency of $f_{p}=10 \mathrm{~Hz}$. This frequency is only a few tens lower than the typical human kinesthetic sense bandwidth [30].

Figure 3 depicts the Bode magnitude plot of $Z_{t}(s)$ for different delays $\left(T_{k}=10^{k-5}[\mathrm{~s}]\right)$ and different wave impedance values $\left(B_{k}=10^{k-1}[\mathrm{~N} \cdot \mathrm{s} / \mathrm{m}]\right)$, when $Z_{e}(s)=0$ and $k \epsilon$ $\{1,2,3,4\}$. Ideally, $Z_{t}(s)$ should be equal to $Z_{e}(s)$ and its magnitude in $\mathrm{dB}$ should diverge to $-\infty$. However, note from Figure 3(a) that $Z_{t}(s)$ is lower bounded for all frequencies and 


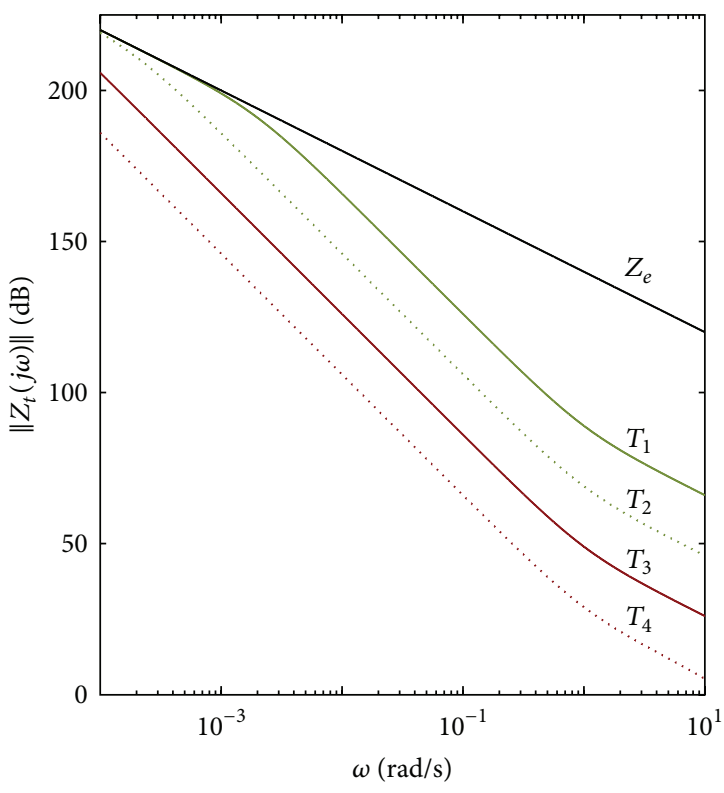

(a) $\left\|Z_{t}(j \omega)\right\|$ as a function of $T$

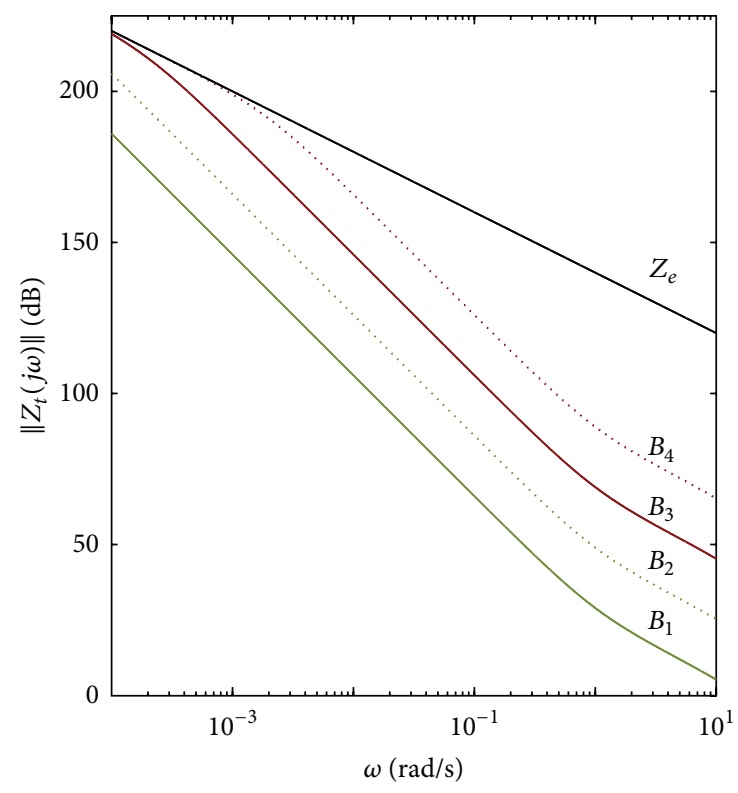

(b) $\left\|Z_{t}(j \omega)\right\|$ as a function of $B$

FIGURE 4: Bode magnitude plot of transmitted impedance $Z_{t}(s)$ for the constrained motion case, $Z_{e}(s)=k_{e} /(\Lambda+s)$. (a) $\left\|Z_{t}(j \omega)\right\|$ plotted for different delays when $B=1[\mathrm{~N} \cdot \mathrm{m} / \mathrm{s}]$ is fixed. (b) $\left\|Z_{t}(j \omega)\right\|$ plotted for different wave impedances when $T=0.1[\mathrm{~s}]$ is fixed.

it increases as the delay increases. Similarly, from Figure 3(b) we can observe that $Z_{t}$ decreases as the wave impedance $B$ decreases. Therefore, lower impedance closer to $Z_{e}$ can be transmitted to the human operator by decreasing the value of the wave impedance.

The transmitted impedance to the operator when the slave robot contacts a hard surface with a stiffness constant of $k_{e}=10^{6}\left[\mathrm{~kg} / \mathrm{s}^{2}\right]$ (i.e., $Z_{e}(s)=k_{e} /(\Lambda+s)$ ) is illustrated in Figure 4. Observe from Figure $4(\mathrm{a})$ that $Z_{t}(s)$ is in general lower than $Z_{e}(s)$ and it continues to decreases as the roundtrip delay increases. Similarly, note from Figure $4(\mathrm{~b})$ that $Z_{t}(s)$ is in general lower than $Z_{e}(s)$ for all values of $B$. It, however, improves as the wave impedance is increased. Therefore, a higher wave impedance enhances the transparency of the system when the slave robot is in contact with a stiff environment.

5.2. Tuning the Wave Impedance. The previous frequency analysis suggests the use of arbitrarily small wave impedance when the slave robot is free to move and an arbitrarily large wave impedance when the slave robot is in contact with a hard environment. In practice, this requires us to alternate $B_{i}$ between a relatively small value $B_{\min }$ and a relatively large value $B_{\max }$. To this end, we propose the update law for the diagonal $j j$ th entry of the wave impedance matrix to be given as

$$
\dot{B}_{s}^{j j}(t)= \begin{cases}\bar{\beta}^{j}(t), & \text { if }\left\|f_{s}^{j}(t)\right\|>0 \\ -\underline{\beta}^{j}(t), & \text { otherwise, }\end{cases}
$$

where $f_{s}^{j}$ is the $j$ th component of $\mathbf{f}_{s}$ and $\bar{\beta}^{j}$ and $\underline{\beta}^{j}$ are nonnegative, bounded scalar functions that drive $B_{s}^{j j}$ to $B_{\max }^{j j}$ and $B_{\min }^{j j}$, respectively. The motivation behind (25) is to smoothly drive the wave impedance toward its two ideal values. In simulations it was observed that faster transitions (or larger jumps) may end up adding unwanted, stable oscillations to the transient response (see Section 7).

\section{Stability Analysis}

The two foremost goals of a bilateral teleoperation control system are to guarantee closed-loop stability and to provide a satisfactory level of transparency regardless of communication delays. Accordingly, we now show the effectiveness of the proposed control law in guaranteeing both objectives under different scenarios. First, we prove closed-stability of the system under time-constant and time-varying communication delays when the environment and the human operator are assumed to be passive systems. Then, we relax the assumption on the human operator by considering the interaction of the slave robot with a hard environment.

Proposition 3. Consider the teleoperation system in (1) with control law (7), (11), and (14). Let $T_{m}(t)=T_{m}$ and $T_{s}(t)=T_{s}$ be constant and assume that the human and remote environment are passive with respect to $\mathbf{r}_{i}$, that is, $\exists \kappa_{i} \in \Re$ such that

$$
-\int_{0}^{t} \mathbf{f}_{i}^{T} \mathbf{r}_{i} d \theta \geq-\kappa_{i}^{2}, \quad \text { for } i=\{m, s\} .
$$

Then, the closed-loop teleoperation system is stable, all signals are bounded, and the system achieves position coordination and static force reflection in the sense of (4) and (5). 
Proof. Let $\mathbf{x}=\left[\mathbf{q}_{m}^{T}, \mathbf{q}_{s}^{T}, \mathbf{r}_{m}^{T}, \mathbf{r}_{s}^{T}, \mathbf{f}_{m}^{T}, \mathbf{f}_{s}^{T}\right]^{T}$ and define $\mathbf{x}_{t}=\mathbf{x}(t+$ $\theta) \in \mathscr{C}, \theta \in\left[-T_{m}-T_{s}, 0\right]$. Consider the following positive definite function:

$$
\begin{aligned}
V\left(t, \mathbf{x}_{t}\right)= & \mathscr{H}_{m}+\mathscr{H}_{s}+\kappa_{m}^{2}+\kappa_{s}^{2}-\int_{0}^{t}\left(\mathbf{f}_{m}^{T} \mathbf{r}_{m}+\mathbf{f}_{s}^{T} \mathbf{r}_{s}\right) d \theta \\
& -\int_{0}^{t}\left(\boldsymbol{\tau}_{m}^{T} \mathbf{r}_{m d}+\boldsymbol{\tau}_{s}^{T} \mathbf{r}_{s d}\right) d \theta
\end{aligned}
$$

where $\mathscr{H}_{i}=(1 / 2) \mathbf{r}_{i}^{T} M_{i}\left(\mathbf{q}_{i}\right) \mathbf{r}_{i}+(1 / 2) \mathbf{q}_{i}^{T} \Lambda K_{i} \mathbf{q}_{i} \geq 0$ for $i \epsilon$ $\{m, s\}$. Note that $\mathbf{r}_{m d}, \boldsymbol{\tau}_{m}, \mathbf{r}_{s d}$, and $\boldsymbol{\tau}_{m}$ are all functions of $\mathbf{r}_{m}$ and $\mathbf{r}_{s}$. Taking the time derivative of (27) we obtain

$$
\begin{aligned}
\dot{V}\left(t, \mathbf{x}_{t}\right)= & \mathbf{f}_{m}^{T} \mathbf{r}_{m}+\boldsymbol{\tau}_{m}^{T} \mathbf{r}_{m}-\dot{\mathbf{q}}_{m}^{T} K_{m} \dot{\mathbf{q}}_{m}+\mathbf{f}_{s}^{T} \mathbf{r}_{s}+\boldsymbol{\tau}_{s}^{T} \mathbf{r}_{s} \\
& -\dot{\mathbf{q}}_{s}^{T} K_{s} \dot{\mathbf{q}}_{s}-\mathbf{f}_{m}^{T} \mathbf{r}_{m}-\mathbf{f}_{s}^{T} \mathbf{r}_{s}-\boldsymbol{\tau}_{m}^{T} \mathbf{r}_{m d}-\boldsymbol{\tau}_{s}^{T} \mathbf{r}_{s d} \\
= & -\left(\mathbf{r}_{m d}-\mathbf{r}_{m}\right)^{T} B_{m}\left(\mathbf{r}_{m d}-\mathbf{r}_{m}\right)-\dot{\mathbf{q}}_{m}^{T} K_{m} \dot{\mathbf{q}}_{m} \\
& -\left(\mathbf{r}_{s d}-\mathbf{r}_{s}\right)^{T} B_{s}\left(\mathbf{r}_{s d}-\mathbf{r}_{s}\right)-\dot{\mathbf{q}}_{s}^{T} K_{s} \dot{\mathbf{q}}_{s} \leq 0 .
\end{aligned}
$$

Since $\dot{V}\left(t, \mathbf{x}_{t}\right) \leq 0$ for all $\mathbf{x}_{t}$, we can conclude that the closed-loop teleoperation system is stable and that $V\left(t, \mathbf{x}_{t}\right) \leq$ $V\left(0, \mathbf{x}_{t}\right)<\infty$, which implies that $\mathbf{r}_{m}, \mathbf{r}_{s}, \mathbf{q}_{s}$, and $\mathbf{q}_{m}$ are bounded. The latter also implies that $\dot{\mathbf{q}}_{m}$ and $\dot{\mathbf{q}}_{s}$ are bounded. Moreover, from the fact that $\int_{0}^{t} \dot{V}\left(\phi, \mathbf{x}_{\phi}\right) d \phi \leq \infty$, we conclude that $\dot{\mathbf{q}}_{m}, \dot{\mathbf{q}}_{s} \in \mathscr{L}_{2}$ and $\boldsymbol{\tau}_{i} \in \mathscr{L}_{\infty}$ (where we applied the fact that $M_{i}, C_{i}, \mathbf{g}_{i}$, and $B_{i}$ are all bounded). Consequently, we obtain that $\ddot{\mathbf{q}}_{i} \in \mathscr{L}_{\infty}$. Then, using Barbalat's lemma [31] we can conclude that $\dot{\mathbf{q}}_{i}(t) \rightarrow \mathbf{0}$ as $t \rightarrow \infty$. Similarly, by using similar arguments, we can show that $\dot{\mathbf{r}}_{m}$ and $\dot{\mathbf{r}}_{s}$ are bounded and that $\mathbf{r}_{s d}(t)-\mathbf{r}_{s}(t)=(1 / 2)\left(\mathbf{r}_{m}\left(t-T_{m}\right)-\mathbf{r}_{s}(t)\right)$ belongs to $\mathscr{L}_{2}$. Therefore, we can apply Barbalat's lemma and conclude that $\mathbf{r}_{m}\left(t-T_{m}\right)-\mathbf{r}_{s}(t) \rightarrow \mathbf{0}$. Since $\dot{\mathbf{q}}_{i} \rightarrow \mathbf{0}$ and $T_{m}$ is finite, we can conclude that $\mathbf{q}_{m}(t)-\mathbf{q}_{s}(t) \rightarrow \mathbf{0}$ as $t \rightarrow \mathbf{0}$.

In order to prove the last statement, let us consider the system in steady-state condition, that is, $\ddot{\mathbf{q}}_{i}(t)=\dot{\mathbf{q}}_{i}(t)=\mathbf{0}$ and $B_{m}(t)=B_{s}(t)$. Then, we have that (8) reduces to

$$
2 \mathbf{f}_{m}=-B_{m} \Lambda\left(\mathbf{q}_{s}-\mathbf{q}_{m}\right), \quad 2 \mathbf{f}_{s}=-B_{s} \Lambda\left(\mathbf{q}_{m}-\mathbf{q}_{s}\right)
$$

which yields that $\mathbf{f}_{m}=-\mathbf{f}_{s}$ and the proof is complete.

The above proposition shows that if the human operator and the environment are modeled as passive systems, the coupling of the overall system is stable and the teleoperator achieves position and force tracking. Note that the result does not impose restrictions on $B_{i}$ other than being positive and finite. In the next statement, we will relax the positive condition on $K_{i}$ by restricting the update rate of $B_{i}$.

Proposition 4. Let $K_{i}=0$ and let $B_{i}$ be governed by (25), with $\dot{B}_{s}(t)<\Lambda B_{s}(t)$ for all $t$. Then, Proposition 3 holds.

Proof. The proof follows similar to the one presented in [19] for Theorem 4.1. It requires the construction of a different Lyapunov function with $B_{m}$ as an state variable.

Let us now generalize the above results to the case of timevarying delays.
Proposition 5. Consider the teleoperation system in (1) with control law governed by (7), (11), (14), (21), and (25). Suppose that $T_{m}(t)$ and $T_{s}(t)$ are time-varying and finite. Furthermore, assume that the human and remote environment are passive with respect to $\mathbf{r}_{i}$, that is, $\exists \kappa_{i} \in \mathfrak{R}$ such that (26) holds. Then, the closed-loop teleoperation system is stable, the coordination error is bounded, and the velocities converge to zero.

Proof. Consider the Lyapunov candidate function in (27). Taking its time derivative yields (28), from which we can conclude (using same arguments as in the proof of Proposition 3) that the closed-loop system is stable, that all signals are bounded, and that $\dot{\mathbf{q}}_{i}, \mathbf{r}_{m}-\mathbf{r}_{m d}$, and $\mathbf{r}_{s}-\mathbf{r}_{s d}$ converge to zero.

Proposition 6. Assume that $\beta^{j}(t), \bar{\beta}^{j}(t) \leq c, T_{m}(t)+T_{s}(t) \leq$ $T$, and $\left|\dot{T}_{i}\right| \leq d$ for some $c, T, d \geq 0$. Then, the coordination error converges to a ball of radius $\left(c T / B_{\min }\right)\left\|\mathbf{q}_{m}\right\|$. Moreover, if $\exists t_{\star}$ such that $\gamma_{m}(t)=\gamma_{s}(t)=1$ for all $t \geq t_{\star}$, then, the system achieves position coordination and static force reflection in the sense of (4) and (5).

Proof. Consider (27) and its time-derivative (28). Since $\int_{0}^{t} \dot{V}\left(\phi, \mathbf{x}_{\phi}\right) d \phi \leq \infty$, we have that $2\left(\mathbf{r}_{m d}(t)-\mathbf{r}_{m}(t)\right)=$ $\gamma_{m}(t) r_{s}\left(t-T_{s}(t)\right)-\mathbf{r}_{m}(t)$ and $2\left(\mathbf{r}_{s d}(t)-\mathbf{r}_{s}(t)\right)=\gamma_{s}(t) \Gamma(t) r_{m}(t-$ $\left.T_{m}(t)\right)-\mathbf{r}_{s}(t)$ belong to $\mathscr{L}_{2}$, where we used (16) and (15). From Proposition 5, we also have that $\mathbf{q}_{i}, \dot{\mathbf{q}}_{i}$, and $\ddot{\mathbf{q}}_{i}$ are bounded, which, together with $\left|\dot{T}_{i}\right| \leq d$, implies that $\dot{\gamma}_{i}, \dot{\mathbf{r}}_{i d}$, and $\dot{\mathbf{r}}_{i}$ are all bounded. Using then Barbalat's lemma, we can conclude that $\mathbf{r}_{m}-\mathbf{r}_{m d}$ and $\mathbf{r}_{s}-\mathbf{r}_{s d}$ converge to zero. Since $\dot{\mathbf{q}}_{i} \rightarrow \mathbf{0}$ (from Proposition 5), we also obtain that $\gamma_{m}(t) \mathbf{q}_{s}\left(t-T_{s}(t)\right)-$ $\mathbf{q}_{m}(t) \rightarrow \mathbf{0}$ and $\gamma_{s}(t) \Gamma(t) \mathbf{q}_{m}\left(t-T_{m}(t)\right)-\mathbf{q}_{s}(t) \rightarrow \mathbf{0}$. Given that $T_{i}$ are finite, we finally have that $\gamma_{m} \mathbf{q}_{s} \rightarrow \mathbf{q}_{m}$ and $\gamma_{s} \Gamma \mathbf{q}_{m} \rightarrow$ $\mathbf{q}_{s}$.

Now, let us assume that the energy filters (22) are initialized at a nonzero value. This assumption does not violate the passivity of the communication channel according to Definition 1. Then, we have that $\gamma_{i} \in(0,1]$. Similarly, note that $0 \leq \Gamma(t) \leq 1+c T / B_{\min }$ for all $t$, since both $\dot{B}_{s}$ and $T_{i}$ are bounded. Using the latter two statements yields that either $\mathbf{q}_{m}(t) \rightarrow \mathbf{q}_{s}(t) \rightarrow \mathbf{0}$, which implies (4), or that $\gamma_{m}(t) \gamma_{s}(t) \Gamma(t) \rightarrow 1$, which implies that $\mathbf{q}_{m}(t)-\mathbf{q}_{s}(t)$ converges to a ball of radius $\left(c T / B_{\min }\right)\left\|\mathbf{q}_{m}\right\|$. Hence, the proof for the first statement is complete.

To prove the second statement, let us assume that $\gamma_{m}(t)=$ $\gamma_{s}(t)=1$ for all $t \geq t_{\star}$. Then, from the previous conclusion, we obtain that $\mathbf{q}_{s}(t)=\gamma_{m}(t) \mathbf{q}_{s}(t) \rightarrow \mathbf{q}_{m}(t)$ as $t \rightarrow \infty$, which implies position coordination in the sense of (4). Similarly, since steady-state conditions imply that $\ddot{\mathbf{q}}_{i}(t), \dot{\mathbf{q}}_{i}(t) \rightarrow \mathbf{0}$, and $B_{m}(t) \rightarrow B_{s}(t)$, we can show that (8) reduces to

$$
2 \mathbf{f}_{m}=-B_{m} \Lambda\left(\mathbf{q}_{s}-\mathbf{q}_{m}\right), \quad 2 \mathbf{f}_{s}=-B_{s} \Lambda\left(\mathbf{q}_{m}-\mathbf{q}_{s}\right)
$$

which yields that $\mathbf{f}_{m}=-\mathbf{f}_{s}$ and the proof is complete.

Up to now, we have shown the stability of the teleoperation system when both human and environment are modeled as passive systems. We also showed that the coordination error between master and slave robots converges to zero if 
(1) the time-delay is constant or if (2) the time delay is timevarying and $\exists t_{\star}$ such that $\gamma_{i}(t)=1$ for all $t \geq t_{\star}$. The latter condition implies that $E_{i}(t) \geq \beta_{i}$ for all $t \geq t_{\star}$. Therefore, let us examine the behavior of the energy reservoirs in (22).

In general, we have that the delayed wave variables are compressed during periods for which the delay decreases and stretched during periods for which the delay increases. Therefore, since $0 \geq \gamma_{i} \geq 1$, the energy reservoirs (22) will increase/decrease when the delay decreases/increases. However, as soon as an energy reservoir decreases below its threshold value $\beta_{i}$, the scale factor $\gamma_{i}$ goes below unity attenuating the energy extracted from the energy reservoir. This means that the proposed energy control strategy tends to favor the buildup of stored energy in the communication channel. In practice, this strategy leads to the buildup of sufficiently large energy such that $E_{i} \geq \beta_{i}$ for all future time.

Let us now relax the assumption of passivity on the human operator by considering the interaction of the slave robot with a hard surface. In the following, we will model the human operator's and environmental forces as

$$
\mathbf{f}_{m}=\boldsymbol{\eta}_{m}-\rho_{m}^{2} \mathbf{r}_{m}, \quad \mathbf{f}_{s}=-\rho_{s}^{2} \mathbf{r}_{s}
$$

where $\rho_{i}$ are constants and $\boldsymbol{\eta}_{m}$ is an arbitrary bounded force, that is, $\exists \eta \in(0, \infty)$ such that $\left\|\boldsymbol{\eta}_{m}(t)\right\| \leq \eta$ for all $t \geq 0$. The human operator's model simulates a nonpassive system where the human exerts a bounded force on the master robot but his/her action is resisted by his/her own passive dynamic component $-\rho_{m}^{2} \mathbf{r}_{m}$. The environment is modeled as a strictly passive system (e.g., a hard surface) with $\rho=\rho_{s}$.

Proposition 7 (see [21]). Consider the teleoperation system in (1) with control law governed by (7), (11), (14), (21), and (25). Let the human operator and environment be modeled as in (31). Assume that $T_{m}(t)$ and $T_{s}(t)$ are time-varying and finite. Then, the system is input-to-state stable [31] and the positions and velocities are uniformly ultimately bounded.

Proof. Let $\mathbf{x}=\left[\dot{\mathbf{q}}_{m}^{T}, \dot{\mathbf{q}}_{s}^{T}, \mathbf{r}_{m}^{T}, \mathbf{r}_{s}^{T}\right]^{T}$ and $\mathbf{y}=\left[\mathbf{q}_{m}^{T}, \mathbf{q}_{s}^{T}, \mathbf{r}_{m}^{T}, \mathbf{r}_{s}^{T}\right]^{T}$. Both vectors are related through a linear diffeomorphism, that is, $\mathbf{x}=\mathscr{T} \mathbf{y}$, where $\mathscr{T} \in \mathfrak{R}^{4 n \times 4 n}$ is a nonsingular, bounded matrix [32]. Therefore, $\mathbf{x}=\mathbf{0} \Leftrightarrow \mathbf{y}=\mathbf{0}$ and $\mathbf{x} \in \mathscr{L}_{\infty} \Leftrightarrow \mathbf{y} \epsilon$ $\mathscr{L}_{\infty}$. Note also that $\mathbf{r}_{m d}, \boldsymbol{\tau}_{m}, \mathbf{r}_{s d}$, and $\boldsymbol{\tau}_{m}$ are all functions of $\mathbf{r}_{m}$ and $\mathbf{r}_{s}$.

Now, define $\mathbf{x}_{t}=\mathbf{x}(t+\theta) \in \mathscr{C}, \theta \in[-T, 0]$ and consider the following function:

$$
V\left(t, \mathbf{x}_{t}\right)=\mathscr{H}_{m}+\mathscr{H}_{s}-\int_{0}^{t}\left(\boldsymbol{\tau}_{m}^{T} \mathbf{r}_{m d}+\boldsymbol{\tau}_{s}^{T} \mathbf{r}_{s d}\right) d \theta
$$

which is positive for all $\mathbf{x}_{t} \neq \mathbf{0}$ and radially unbounded. Taking its time derivative yields

$$
\begin{aligned}
\dot{V}\left(t, \mathbf{x}_{t}\right)= & \boldsymbol{\eta}_{m}^{T} \mathbf{r}_{m}-\rho_{m}^{2} \mathbf{r}_{m}^{T} \mathbf{r}_{m}-\rho_{s}^{2} \mathbf{r}_{s}^{T} \mathbf{r}_{s} \\
& -\dot{\mathbf{q}}_{s}^{T} K_{s} \dot{\mathbf{q}}_{s}-\left(\mathbf{r}_{m d}-\mathbf{r}_{m}\right)^{T} B_{m}\left(\mathbf{r}_{m d}-\mathbf{r}_{m}\right) \\
& -\dot{\mathbf{q}}_{m}^{T} K_{m} \dot{\mathbf{q}}_{m}-\left(\mathbf{r}_{s d}-\mathbf{r}_{s}\right)^{T} B_{s}\left(\mathbf{r}_{s d}-\mathbf{r}_{s}\right)
\end{aligned}
$$

which can be bounded by

$$
\begin{aligned}
\dot{V}\left(t, \mathbf{x}_{t}\right) \leq & \eta\left\|\mathbf{r}_{m}\right\|-\rho_{m}^{2}\left\|\mathbf{r}_{m}\right\|^{2}-\rho_{s}^{2}\left\|\mathbf{r}_{s}\right\|^{2} \\
& -\underline{\sigma}\left(K_{m}\right)\left\|\dot{\mathbf{q}}_{m}\right\|^{2}-\underline{\sigma}\left(K_{s}\right)\left\|\dot{\mathbf{q}}_{s}\right\|^{2} .
\end{aligned}
$$

Next, define $\epsilon=\min \left\{\rho_{m}^{2}, \rho_{s}^{2}, \underline{\sigma}\left(K_{m}\right), \underline{\sigma}\left(K_{s}\right)\right\}$ and let $\epsilon_{0} \in(0, \epsilon)$ be an arbitrarily small positive constant. We can then upper bound (34) as $\dot{V}\left(t, \mathbf{x}_{t}\right) \leq \eta\left\|\mathbf{x}_{t}\right\|-\left(\epsilon-\epsilon_{0}\right)\left\|\mathbf{x}_{t}\right\|^{2}-\epsilon_{0}\left\|\mathbf{x}_{t}\right\|^{2}$ and obtain that

$$
\dot{V}\left(t, \mathbf{x}_{t}\right) \leq-\epsilon_{0}\left\|\mathbf{x}_{t}\right\|^{2}, \quad \forall\left\|\mathbf{x}_{t}\right\| \geq \frac{\eta}{\epsilon-\epsilon_{0}} .
$$

Therefore, we can conclude that the system, with state variable $\mathbf{x}_{t}$, is input-to-state stable with ultimate bound given by $\eta /(\epsilon-$ $\left.\epsilon_{0}\right)$. Since boundedness of $\mathbf{x}$ implies boundedness of $\mathbf{y}$, we also conclude that the positions and velocities are uniformly ultimately bounded.

Next, we will evaluate the case where the human operator exerts a bounded force without a passive component, that is, $\rho_{m}=0$. Essentially, this exemplifies any admissible scenario for the human operator.

Proposition 8. Consider the teleoperation system in (1) with control law governed by (7), (11), (14), (21), and (25). Let the human operator and environment be modeled as in (31) for $\rho_{m}=0$. Assume that $T_{m}(t)$ and $T_{s}(t)$ are time-varying and finite and that $\dot{T}_{s} \in(-\infty, 1)$. Then, the system is input-to-state stable and the positions and velocities are uniformly ultimately bounded.

Proof. Suppose that $\exists \underline{\delta} \leq \bar{\delta}<1$ such that $\underline{\delta} \leq \dot{T}_{s}(t) \leq \bar{\delta}$ for all $t \geq 0$ and consider the following positive definite, radially unbounded function:

$$
\begin{aligned}
V\left(t, \mathbf{x}_{t}\right)= & \mathscr{H}_{m}+\mathscr{H}_{s}+\rho_{0}^{2} \int_{t-T_{s}(t)}^{t} \mathbf{r}_{s}^{T} \mathbf{r}_{s} d \theta \\
& -\int_{0}^{t}\left(\boldsymbol{\tau}_{m}^{T} \mathbf{r}_{m d}+\boldsymbol{\tau}_{s}^{T} \mathbf{r}_{s d}\right) d \theta,
\end{aligned}
$$

where $\rho_{0}^{2}=\rho_{s}^{2} /(1-\underline{\delta})-\rho_{1}^{2}>0$ for some small enough $\rho_{1}>0$ (note that such $\rho_{0}$ and $\rho_{1}$ exist as long as $\dot{T}_{s} \in(-\infty, 1)$ ). The time-derivative of (36) can be computed as

$$
\begin{aligned}
\dot{V}\left(t, \mathbf{x}_{t}\right)= & \mathbf{f}_{m}^{T} \mathbf{r}_{m}+\boldsymbol{\tau}_{m}^{T} \mathbf{r}_{m}-\dot{\mathbf{q}}_{m}^{T} K_{m} \dot{\mathbf{q}}_{m}+\mathbf{f}_{s}^{T} \mathbf{r}_{s}+\boldsymbol{\tau}_{s}^{T} \mathbf{r}_{s} \\
& +\rho_{0}^{2}\left(1-\dot{T}_{s}\right)\left(\mathbf{r}_{s}^{T} \mathbf{r}_{s}-\mathbf{r}_{s}^{T}\left(t-T_{s}\right) \mathbf{r}_{s}\left(t-T_{s}\right)\right) \\
& -\dot{\mathbf{q}}_{s}^{T} K_{s} \dot{\mathbf{q}}_{s}-\boldsymbol{\tau}_{m}^{T} \mathbf{r}_{m d}-\boldsymbol{\tau}_{s}^{T} \mathbf{r}_{s d}
\end{aligned}
$$

which after some manipulation can be bounded as

$$
\begin{aligned}
\dot{V}\left(t, \mathbf{x}_{t}\right) \leq & \boldsymbol{\eta}_{m}^{T} \mathbf{r}_{m}-\dot{\mathbf{q}}_{m}^{T} K_{m} \dot{\mathbf{q}}_{m}-\rho_{1}^{2} \mathbf{r}_{s}^{T} \mathbf{r}_{s}-\dot{\mathbf{q}}_{s}^{T} K_{s} \dot{\mathbf{q}}_{s} \\
& -\rho_{2}^{2} \mathbf{r}_{s}^{T}\left(t-T_{s}\right) \mathbf{r}_{s}\left(t-T_{s}\right) \\
& -\left(\mathbf{r}_{m d}-\mathbf{r}_{m}\right)^{T} B_{m}\left(\mathbf{r}_{m d}-\mathbf{r}_{m}\right) \\
& -\left(\mathbf{r}_{s d}-\mathbf{r}_{s}\right)^{T} B_{s}\left(\mathbf{r}_{s d}-\mathbf{r}_{s}\right)
\end{aligned}
$$




$$
\begin{aligned}
& \leq \eta\left\|\mathbf{r}_{m}\right\|-\underline{\sigma}\left(K_{m}\right)\left\|\dot{\mathbf{q}}_{m}\right\|^{2}-\rho_{1}^{2}\left\|\mathbf{r}_{s}^{T}\right\|^{2} \\
& -\rho_{2}^{2}\left\|\mathbf{r}_{s}^{T}\left(t-T_{s}\right)\right\|^{2}-\underline{\sigma}\left(K_{s}\right)\left\|\dot{\mathbf{q}}_{s}\right\|^{2} \\
& -\frac{\sigma\left(B_{m}\right)}{4}\left\|\gamma_{m}(t) \mathbf{r}_{s}\left(t-T_{s}\right)-\mathbf{r}_{m}\right\|^{2},
\end{aligned}
$$

where $\rho_{2}^{2}=\rho_{0}^{2}(1-\bar{\delta}) \leq \rho_{0}^{2}\left(1-\dot{T}_{s}\right)$ is a positive constant. Now, using (16) and combining terms we obtain that

$$
\begin{aligned}
\dot{V}\left(t, \mathbf{x}_{t}\right) \leq & \eta\left\|\mathbf{r}_{m}\right\|-\underline{\sigma}\left(K_{m}\right)\left\|\dot{\mathbf{q}}_{m}\right\|^{2}-\rho_{1}^{2}\left\|\mathbf{r}_{s}^{T}\right\|^{2} \\
& -\rho_{2}^{2}\left(1-\gamma_{m}^{2}\right)\left\|\mathbf{r}_{s}^{T}\left(t-T_{s}\right)\right\|^{2}-\underline{\sigma}\left(K_{s}\right)\left\|\dot{\mathbf{q}}_{s}\right\|^{2} \\
& -\left\|c_{1} \mathbf{r}_{s}\left(t-T_{s}\right)-c_{2} \mathbf{r}_{m}\right\|^{2}-\left(\frac{1}{4} \underline{\sigma}\left(B_{m}\right)-c_{2}^{2}\right)\left\|\mathbf{r}_{m}\right\|^{2} \\
\leq & \eta\left\|\mathbf{r}_{m}\right\|-\underline{\sigma}\left(K_{m}\right)\left\|\dot{\mathbf{q}}_{m}\right\|^{2}-\rho_{1}^{2}\left\|\mathbf{r}_{s}^{T}\right\|^{2} \\
& -\underline{\sigma}\left(K_{s}\right)\left\|\dot{\mathbf{q}}_{s}\right\|^{2}-\left(\frac{1}{4} \underline{\sigma}\left(B_{m}\right)-c_{2}^{2}\right)\left\|\mathbf{r}_{m}\right\|^{2},
\end{aligned}
$$

where

$$
c_{1}=\gamma_{m} \sqrt{\rho_{2}^{2}+\frac{1}{4} \underline{\sigma}\left(B_{m}\right)}, \quad c_{2}=\frac{\underline{\sigma}\left(B_{m}\right)}{4 \sqrt{\rho_{2}^{2}+(1 / 4) \underline{\sigma}\left(B_{m}\right)}} .
$$

If we define $\varepsilon=\min \left\{\rho_{1}^{2},(1 / 4) \underline{\sigma}\left(B_{m}\right)-c_{2}^{2}, \underline{\sigma}\left(K_{m}\right), \underline{\sigma}\left(K_{s}\right)\right\}>0$ and let $\varepsilon_{0} \in(0, \varepsilon)$, we obtain that

$$
\dot{V}\left(t, \mathbf{x}_{t}\right) \leq \eta\left\|\mathbf{x}_{t}\right\|-\left(\varepsilon-\varepsilon_{0}\right)\left\|\mathbf{x}_{t}\right\|^{2}-\varepsilon_{0}\left\|\mathbf{x}_{t}\right\|^{2}
$$

and, consequently, $\dot{V}\left(t, \mathbf{x}_{t}\right) \leq-\varepsilon_{0}\left\|\mathbf{x}_{t}\right\|^{2}$, for all $\left\|\mathbf{x}_{t}\right\| \geq \eta /\left(\varepsilon-\varepsilon_{0}\right)$. The latter implies that the system, with state variable $\mathbf{x}_{t}$, is input-to-state stable with ultimate bound given by $\eta /(\varepsilon-$ $\left.\varepsilon_{0}\right)$. Since boundedness of $\mathbf{x}$ implies boundedness of $\mathbf{y}$, we also conclude that the positions and velocities are uniformly ultimately bounded.

\section{Simulations}

To demonstrate the performance of the proposed bilateral control, we present a simulation example with a set of two identical 2-DOF planar revolute-joint manipulators [10], akin to the pair shown in Figure 1. The nonlinear dynamics of the manipulators (1), with the gravitational torques neglected due to the system's planar configuration, are given by

$$
M_{i}\left(\mathbf{q}_{i}\right)=\left[\begin{array}{cc}
\alpha_{i} & \zeta_{i} \\
\zeta_{i} & \gamma
\end{array}\right], \quad C_{i}\left(\mathbf{q}_{i}, \dot{\mathbf{q}}_{i}\right)=\left[\begin{array}{cc}
\lambda_{i} \dot{q}_{i}^{2} & \lambda_{i}\left(\dot{q}_{i}^{1}+\dot{q}_{i}^{2}\right) \\
-\lambda_{i} \dot{q}_{i}^{1} & 0
\end{array}\right]
$$

for $\alpha_{i}=81.6+9.19 \cos \left(q_{i}^{2}\right)\left[10^{-2} \cdot \mathrm{N} \cdot \mathrm{m}^{2}\right], \zeta_{i}=4.63+$ $4.59 \cos \left(q_{i}^{2}\right)\left[10^{-2} \cdot \mathrm{N} \cdot \mathrm{m}^{2}\right], \gamma=4.63\left[10^{-2} \cdot \mathrm{N} \cdot \mathrm{m}^{2}\right]$, and $\lambda_{i}=-4.59 \sin \left(q_{i}^{2}\right)\left[10^{-2} \cdot \mathrm{kg} \cdot \mathrm{m}^{3}\right]$. The task space coordinates of the manipulators' end-effectors can be obtained from the joint coordinates as $x_{i}=l_{1} \cos \left(q_{i}^{1}\right)+l_{2} \cos \left(q_{i}^{1}+q_{i}^{2}\right)$ and $y_{i}=l_{1} \sin \left(q_{i}^{1}\right)+l_{2} \sin \left(q_{i}^{1}+q_{i}^{2}\right)$ where $\left(x_{i}, y_{i}\right)$ are the Cartesian coordinates of the robots and $l_{1}=0.3[\mathrm{~m}]$ and $l_{2}=l_{1}$ represent the lengths of the robots' first and second links, respectively. The communication delays between master and slave are assumed to be given as $T_{m}(t)=0.5+0.2 \cos (5 t)[\mathrm{s}]$ and $T_{s}(t)=0.5+0.2 \sin (5 t)[\mathrm{s}$ ] for a maximum round-trip delay of $T=1+(\sqrt{2} / 5)[\mathrm{s}]$.

The environment is modeled as a stiff wall located at $x_{w}=$ $-0.075[\mathrm{~m}]$ with a reaction force given by

$$
\begin{aligned}
& f_{w} \\
& = \begin{cases}-2.5 \times 10^{3} \dot{x}_{s}-5 \times 10^{4}\left(x_{s}-x_{w}\right)[\mathrm{N}], & \text { if } x_{s} \leq x_{w} \\
0[\mathrm{~N}], & \text { otherwise. }\end{cases}
\end{aligned}
$$

Since the force is applied at the slave's end-effector, the equivalent environmental torque at the robot's joints can be obtained from $\mathbf{f}_{s}=J^{T}\left(\mathbf{q}_{s}\right)\left[f_{w}, 0\right]^{T}$, where $J^{T}\left(\mathbf{q}_{s}\right)$ is the slave robot's Jacobian matrix [28]. The human operator, on the other hand, is modeled as a bounded P-type controller $f_{m}^{i}=$ sat $_{-25,25}\left\{150\left(q_{d}^{i}-q_{m}^{i}\right)\right\}$ where $\mathbf{q}_{d} \in \mathfrak{R}^{2}$ is the desired jointtrajectory given by

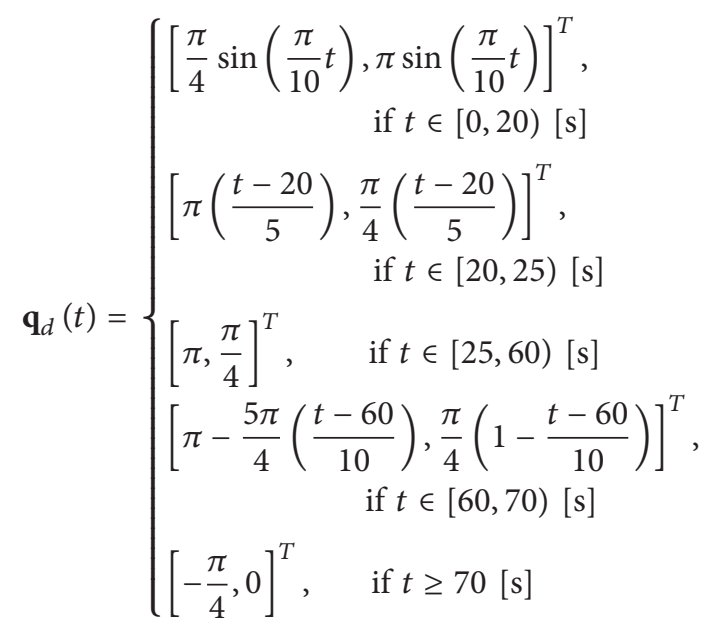

and $\operatorname{sat}_{\underline{x}, \bar{x}}\{x\}$ is the saturation function [31] with $\underline{x}$ and $\bar{x}$ as lower and upper limits. The gains for the controller are $K_{i}=$ $2[\mathrm{~N} / \mathrm{m}], \Lambda=1[1 / \mathrm{s}]$, and $\beta_{i}=0.05[\mathrm{~J}]$. We assume that the desired wave impedance values for the free motion and hardcontact motion scenarios are $B_{\min }^{11}=B_{\min }^{22}=10[\mathrm{~N} \cdot \mathrm{s} / \mathrm{m}]$ and $B_{\max }^{11}=B_{\max }^{22}=250[\mathrm{~N} \cdot \mathrm{s} / \mathrm{m}]$, respectively. We then simulate the response of the teleoperation system with the proposed controller using constant wave impedance equal to $B_{\min }$ and time-varying wave impedance value. For the time-varying wave impedance case, we choose the following update law $\bar{\beta}(t)=0.05\left(B_{\max }-B_{s}(t)\right)+0.3\left(B_{\max }-B_{s}(t)\right)^{1 / 4}+0.02\left(B_{\max }-\right.$ $\left.B_{s}(t)\right)^{3 / 2}$ and $\beta(t)=0.05\left(B_{s}(t)-B_{\min }\right)+0.3\left(B_{s}(t)-B_{\min }\right)^{1 / 4}+$ $0.02\left(B_{s}(t)-\bar{B}_{\min }\right)^{3 / 2}$. 

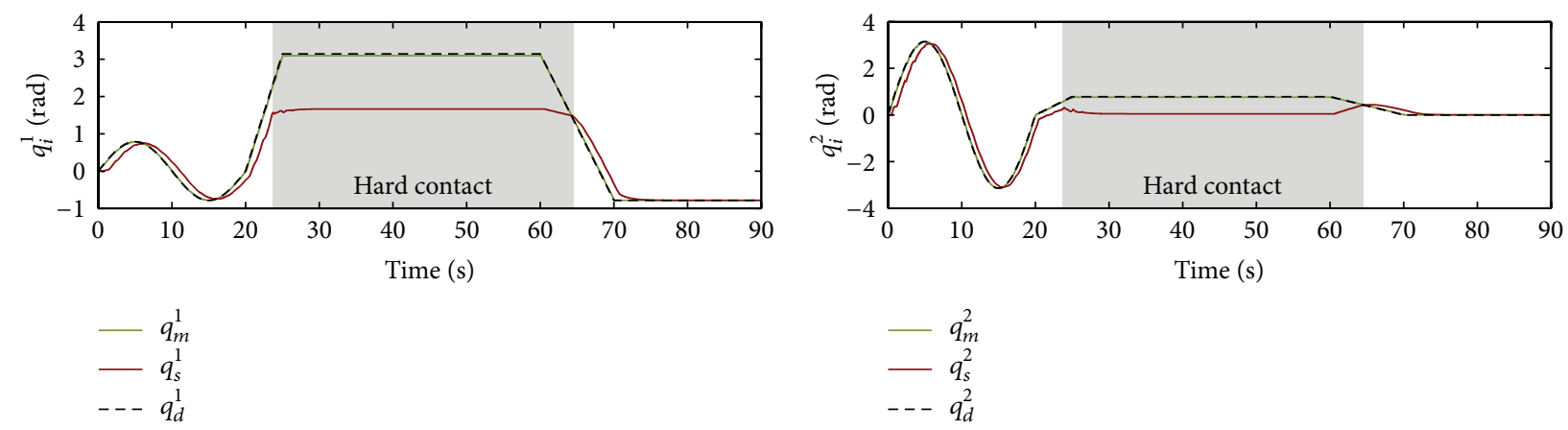

(a) First link position of master and slave robots

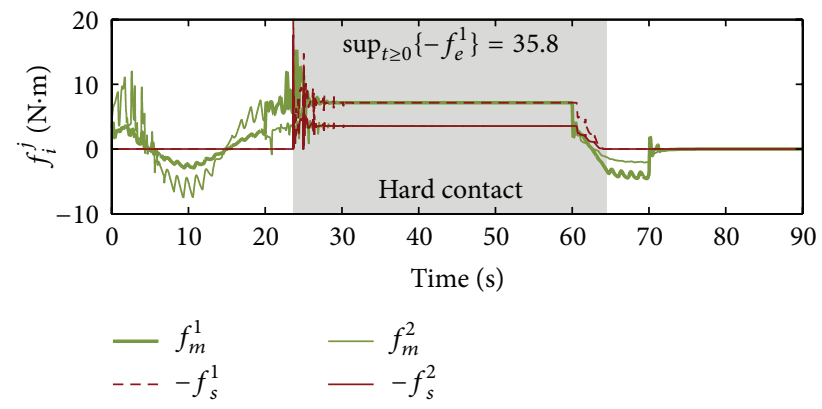

(c) Human and environmental forces

(b) Second link position of master and slave robots

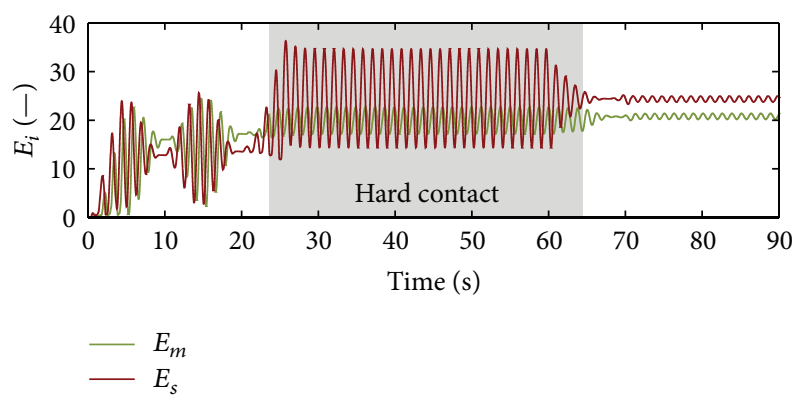

(d) Energy level in reservoirs

FIGURE 5: System response for $B_{m, s}=10[\mathrm{~N} \cdot \mathrm{s} \cdot \mathrm{m}]$.

Figure 5 illustrates the motion, force, and energy stored by the teleoperation system when low constant wave impedance value is used. Observe that the slave robot tracks the motion of the master robot with relatively small error during the free motion section of the experiment (i.e., for $t \epsilon$ $[0,24][\mathrm{s}]$ and $t \geq 64[\mathrm{~s}])$. However, as soon as the slave robot impacts the wall, the master-slave error starts increasing due to the ability of the human operator to still manipulate the master robot with little effort while the position of the slave robot is locked. In fact, note from Figure 5(c) that the torques exerted by the operator during the hard-contact portion of the experiment $(t \in[24,64][s])$ do not exceed $10[\mathrm{~N}$. $\mathrm{m}]$ and do not differ significantly from the torques exerted by the operator during the free motion section. This is an adverse effect of using small wave impedance value when the environmental impedance is high: the operator looses the ability to properly perceived the higher environmental impedance. On the other hand, observe that force reflection is rapidly achieved during the hard-contact experiment and that the passivity-based control properly stores enough energy to guarantee asymptotic position convergence despite time-varying delays (see Figure 5(d)).

Figure 6 illustrates simulation results when the proposed time-varying wave impedance strategy is employed. Observe from Figures 6(a) and 6(b) that the behavior of the teleoperation system is similar to the previous case during the free motion section, as it should be expected due to the use of the same wave impedance value. The main difference occurs during the hard-contact motion scenario. Note that the master-slave error starts decreasing until converging to a relatively small value as the wave impedance value starts increasing (see Figure 7). As seen in Figure 6(c), the human operator is also able to perceive higher resistance during the restricted section of the experiment, properly transmitting higher environmental impedance. This can be interpreted as in improvement on transparency when compared to the previous example. In addition, note that force reflection is eventually achieved and that the proposed controller satisfactorily builds enough energy to guarantee asymptotic position convergence despite time-varying delays (see Figure 6(d)). It is worth mentioning that the transitions between impedance values may exhibit some unwanted behaviors such as largeryet stable-transient oscillations. Therefore, the choice of switching functions (25) needs to be carefully studied.

For sake of comparison, Figure 8 plots the master-slave coordination error for different wave impedance values. Note that our proposed control approach assimilates satisfactorily the ideal behavior of using constant lower impedance while the slave is in free motion and the behavior of using larger impedance once the slave robot makes contact with the wall. Furthermore, note that the position drift during hardcontact decreases by $86 \%$ when using the proposed control strategy.

Likewise, the average transmitted impedance and static stiffness to the human operator while in free and hard-contact motion scenarios, respectively, are illustrated in Figure 9 for 

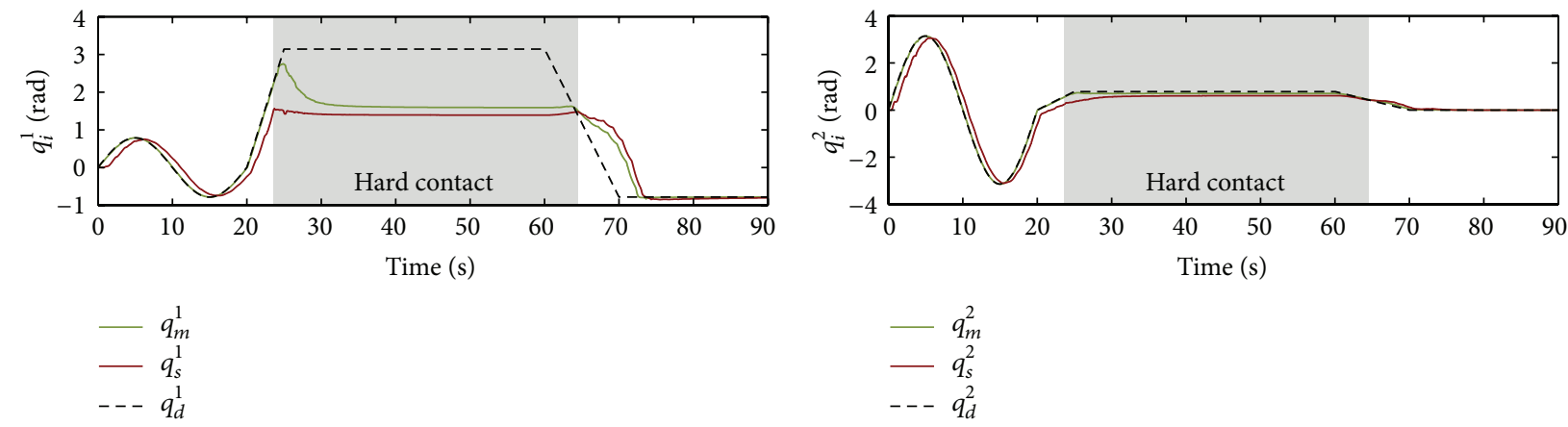

(b) Second link position of master and slave robots

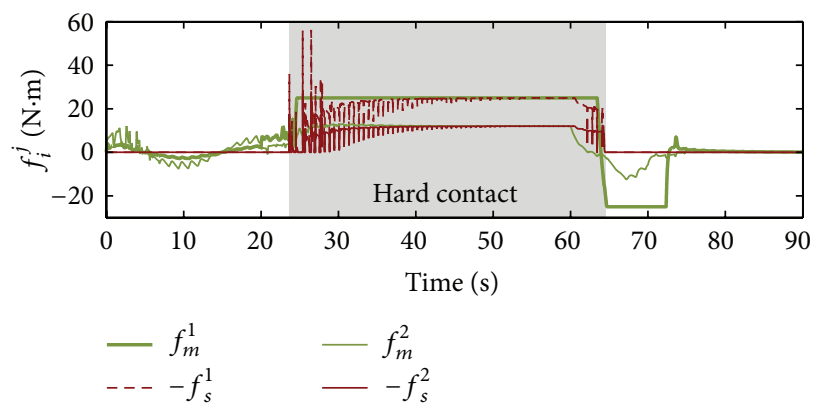

(c) Human and environmental forces

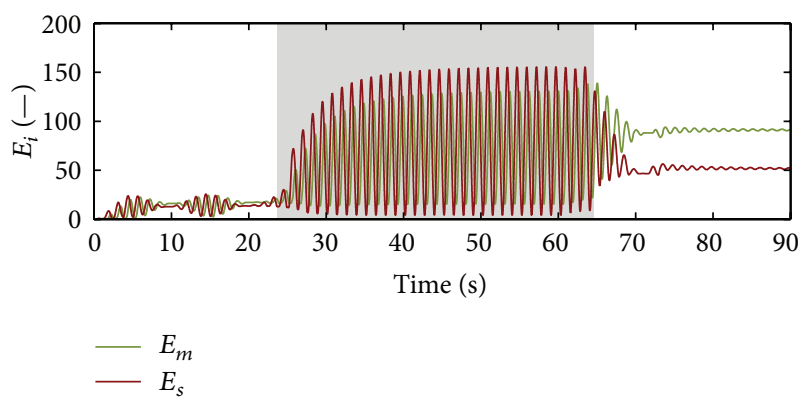

(d) Energy level in reservoirs

FiguRE 6: System response for $B_{m, s}(t) \in[10,250]$.

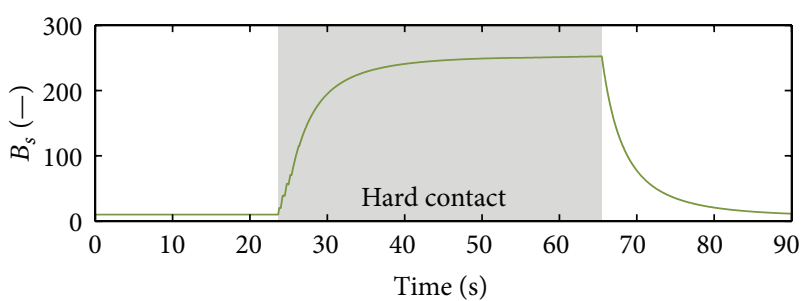

FIGURE 7: Evolution of $B_{s}(t)$.

different $B_{i}$ values. The average impedance $Z_{\text {ave }}$ and average static stiffness $K_{\text {ave }}[10]$ where computed as

$$
\begin{gathered}
Z_{\mathrm{ave}}=\frac{1}{t_{b}-t_{a}} \int_{t_{a}}^{t_{b}} \frac{\left\|\mathbf{f}_{m}\right\|}{\left\|\dot{\mathbf{q}}_{m}\right\|} d t, \\
K_{\mathrm{ave}}=\frac{1}{t_{d}-t_{c}} \int_{t_{c}}^{t_{d}} \frac{\left\|\mathbf{f}_{m}\right\|}{\left\|\mathbf{q}_{m}-\mathbf{q}_{s}\right\|} d t,
\end{gathered}
$$

where $\left[t_{a}, t_{b}\right]=[7,14][\mathrm{s}]$ and $\left[t_{c}, t_{d}\right]=[40,60][\mathrm{s}]$ correspond to intervals of time where $\left\|\dot{\mathbf{q}}_{m}\right\|$ and $\left\|\mathbf{q}_{m}-\mathbf{q}_{s}\right\|$ were nearly constant and did not cross zero. In general, lower values of impedance and larger values of stiffness are desirable. Observe, from Figure 9, that the best overall results are obtained when time-varying wave impedance is employed, with the lowest transmitted impedance values during free motion and a relatively large static stiffness during hard contact.

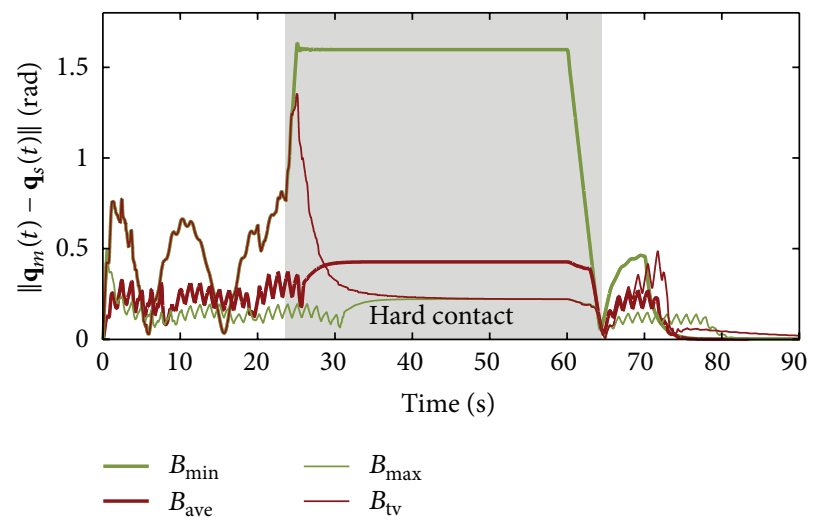

FIGURE 8: Master-slave coordination error for constant wave impedances $B_{\min }=10, B_{\mathrm{ave}}=130$, and $B_{\max }=250$ and time-varying wave impedance $B_{\mathrm{tv}}=[10,250]$.

\section{Conclusion}

One of the main challenges in the realization of timedelayed bilateral teleoperators is how to adjust transparency without compromising closed-loop stability. In this paper, we presented a closed-loop stable wave-based bilateral control architecture that properly adjusts the transparency of the teleoperation system, regardless of the presence of time-varying communication delays. We studied the effect that the wave impedance has on transparency and, based on our findings, we then developed an update policy that smoothly adapts 


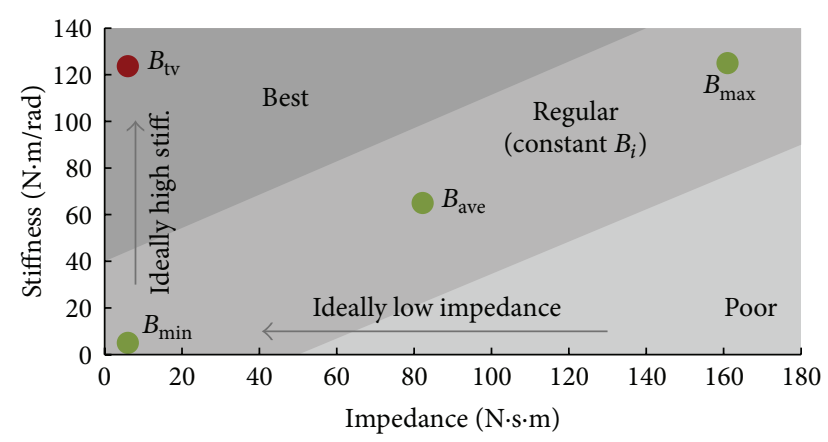

FIGURE 9: Impedance versus static stiffness perceived by the human operator during free motion and hard contact, respectively. Lower values of impedance and higher values of stiffness are desirable.

the wave impedance value according to the current environmental dynamics. In contrast to our previous work [19], the transitions between impedance values can be performed at arbitrarily fast rates. In addition, we proved that the proposed control framework guarantees asymptotic convergence of the velocities and boundedness of the coordination error if the human operator and environment are passive. Then, we relaxed the passivity assumption on the human operator by allowing any bounded human model and showed that the master-slave position error and velocities are ultimately bounded. Furthermore, we illustrated via simulations the effectiveness of the control strategy and compared its performance against the use of different constant wave impedance values under time-varying environmental dynamics. The simulation results demonstrated that the proposed control architecture can transmit more faithfully the environmental impedance value to the human operator. Future research directions include the consideration of more diverse remote dynamics, including nonpassive environments.

\section{Conflict of Interests}

The author declares that there is no conflict of interests regarding the publication of this paper.

\section{Acknowledgment}

The author would like to thank Dr. Mark W. Spong for his insightful comments on previous versions of this work.

\section{References}

[1] D. A. Lawrence, "Stability and transparency in bilateral teleoperation," IEEE Transactions on Robotics and Automation, vol. 9, no. 5, pp. 624-637, 1993.

[2] Y. Yokokohji and T. Yoshikawa, "Bilateral control of masterslave manipulators for ideal kinesthetic coupling - formulation and experiment," IEEE Transactions on Robotics and Automation, vol. 10, no. 5, pp. 605-619, 1994.

[3] K. Hashtrudi-Zaad and S. E. Salcudean, "Analysis of control architectures for teleoperation systems with impedance/ admittance master and slave manipulators," International Journal of Robotics Research, vol. 20, no. 6, pp. 419-445, 2001.

[4] R. W. Daniel and R. R. McAree, "Fundamental limits of performance for force reflecting teleoperation," The International Journal of Robotics Research, vol. 17, no. 8, pp. 811-830, 1998.

[5] F. Kobayashi, G. Ikai, W. Fukui, and F. Kojima, "Two-fingered haptic device for robot hand teleoperation," Journal of Robotics, vol. 2011, Article ID 419465, 8 pages, 2011.

[6] B. Willaert, D. Reynaerts, H. van Brussel, and E. B. V. Poorten, "Bilateral teleoperation: quantifying the requirements for and restrictions of ideal transparency," IEEE Transactions on Control Systems Technology, vol. 22, no. 1, pp. 387-395, 2014.

[7] R. V. Dubey, T. F. Chan, and S. E. Everett, "Variable damping impedance control of a bilateral telerobotic system," IEEE Control Systems Magazine, vol. 17, no. 1, pp. 37-45, 1997.

[8] P. Arcara and C. Melchiorri, "Control schemes for teleoperation with time delay: a comparative study," Robotics and Autonomous Systems, vol. 38, no. 1, pp. 49-64, 2002.

[9] P. F. Hokayem and M. W. Spong, "Bilateral teleoperation: an historical survey," Automatica, vol. 42, no. 12, pp. 2035-2057, 2006.

[10] E. J. Rodríguez-Seda, D. Lee, and M. W. Spong, "Experimental comparison study of control architectures for bilateral teleoperators," IEEE Transactions on Robotics, vol. 25, no. 6, pp. 13041318, 2009.

[11] R. J. Anderson and M. W. Spong, "Bilateral control of teleoperators with time delay," in Proceedings of the IEEE Conference on Decision and Control, pp. 167-173, Austin, Tex, USA, December 1988.

[12] G. Niemeyer and J.-J. E. Slotine, "Stable adaptive teleoperation," IEEE Journal of Oceanic Engineering, vol. 16, no. 1, pp. 152-162, 1991.

[13] D. J. Lee and M. W. Spong, "Passive bilateral teleoperation with constant time delay," IEEE Transactions on Robotics, vol. 22, no. 2, pp. 269-281, 2006.

[14] E. Nuño, R. Ortega, N. Barabanov, and L. Basañez, "A globally stable PD controller for bilateral teleoperators," IEEE Transactions on Robotics, vol. 24, no. 3, pp. 753-758, 2008.

[15] D. Lee and K. Huang, "Passive-set-position-modulation framework for interactive robotic systems," IEEE Transactions on Robotics, vol. 26, no. 2, pp. 354-369, 2010.

[16] S. Hirche and M. Buss, "Human-oriented control for haptic teleoperation," Proceedings of the IEEE, vol. 100, no. 3, pp. 623647, 2012.

[17] P. Arcara and C. Melchiorri, "Comparison and improvement of control schemes for robotic teleoperation systems with time delay," in Advances in Control of Articulated and Mobile Robots, B. Siciliano, O. Khatib, and F. Groen, Eds., vol. 10 of Springer Tracts in Advanced Robotics, pp. 39-60, Springer, Berlin, Germany, 2004.

[18] C. Secchi, S. Stramigioli, and C. Fantuzzi, "Position drift compensation in port-Hamiltonian based telemanipulation," in Proceedings of the IEEE/RSJ International Conference on Intelligent Robots and Systems (IROS '06), pp. 4211-4216, IEEE, Beijing, China, October 2006.

[19] E. J. Rodríguez-Seda and M. W. Spong, "A time-varying wave impedance approach for transparency compensation in bilateral teleoperation," in Proceedings of the IEEE/RSJ International Conference on Intelligent Robots and Systems (IROS '09), pp. 4609-4615, St. Louis, Mo, USA, October 2009. 
[20] N. A. Tanner and G. Niemeyer, "Online tuning of wave impedance in telerobotics," in Proceedings of the IEEE Conference on Robotics, Automation and Mechatronics, pp. 7-12, December 2004.

[21] E. J. Rodríguez-Seda, “Transparency compensation for bilateral teleoperators with communication time-varying delays," in Proceedings of the IEEE/RSJ International Conference on Intelligent Robots and Systems, pp. 4375-4380, September 2014.

[22] M. Franken, S. Stramigioli, S. Misra, C. Secchi, and A. Macchelli, "Bilateral telemanipulation with time delays: a twolayer approach combining passivity and transparency," IEEE Transactions on Robotics, vol. 27, no. 4, pp. 741-756, 2011.

[23] J. Kim, P. H. Chang, and H.-S. Park, “Two-channel transparency-optimized control architectures in bilateral teleoperation with time delay," IEEE Transactions on Control Systems Technology, vol. 21, no. 1, pp. 40-51, 2013.

[24] C. A. López Martínez, R. van de Molengraft, and M. Steinbuch, "High performance teleoperation using switching robust control," in Proceedings of the World Haptics Conference (WHC '13), pp. 383-388, Daejeon, Republic of Korea, April 2013.

[25] N. Chopra, M. W. Spong, and R. Lozano, "Adaptive coordination control of bilateral teleoperators with time delay," in Proceedings of the 43rd IEEE Conference on Decision and Control (CDC '04), pp. 4540-4547, Paradise Island, Bahamas, December 2004.

[26] J. K. Hale, "A stability theorem for functional-differential equations," Proceedings of the National Academy of Sciences of the United States of America, vol. 50, pp. 942-946, 1963.

[27] A. van der Schaft, L2-Gain Stability and Passivity Techniques in Nonlinear Control, Springer, London, UK, 2000.

[28] M. W. Spong, S. Hutchinson, and M. Vidyasagar, Robot Modeling and Control, John Wiley \& Sons, New York, NY, USA, 2006.

[29] S. Hirche and M. Buss, "Human perceived transparency with time delay," in Advances in Telerobotics, M. Ferre, M. Buss, R. Aracil, C. Melchiorri, and C. Balaguer, Eds., Springer Tracts in Advanced Robotics, pp. 191-209, Springer, Berlin, Germany, 2007.

[30] K. S. Hale and K. M. Stanney, "Deriving haptic design guidelines from human physiological, psychophysical, and neurological foundations," IEEE Computer Graphics and Applications, vol. 24, no. 2, pp. 33-39, 2004.

[31] H. K. Khalil, Nonlinear Systems, Prentice Hall, Englewood Cliffs, NJ, USA, 2002.

[32] M. W. Spong, R. Ortega, and R. Kelly, "Comments on 'Adaptive manipulator control: a case study' by J Slotine and W. Li," IEEE Transactions on Automatic Control, vol. 35, no. 6, pp. 761-762, 1990. 

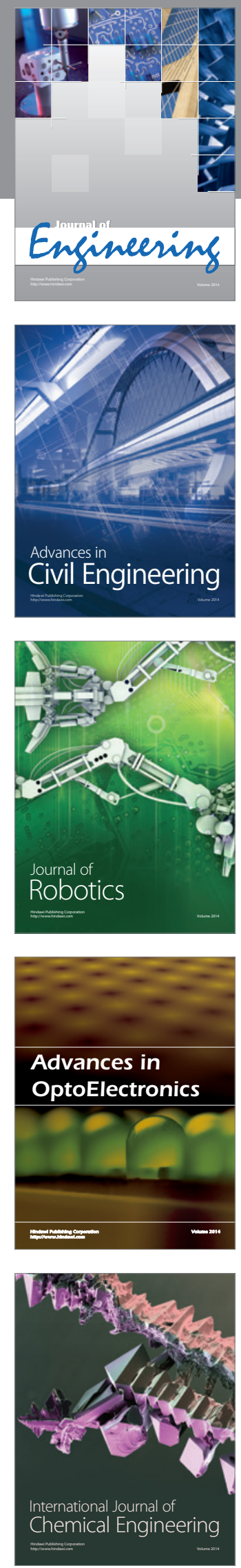

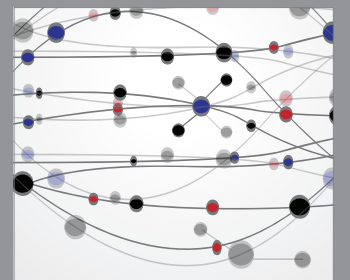

The Scientific World Journal
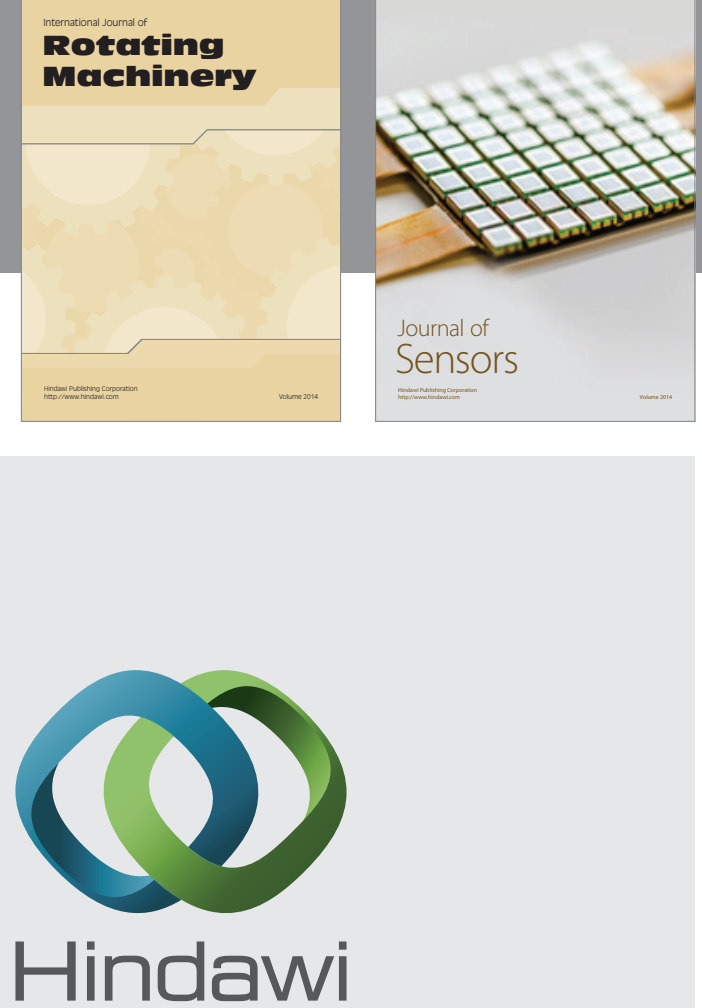

Submit your manuscripts at http://www.hindawi.com
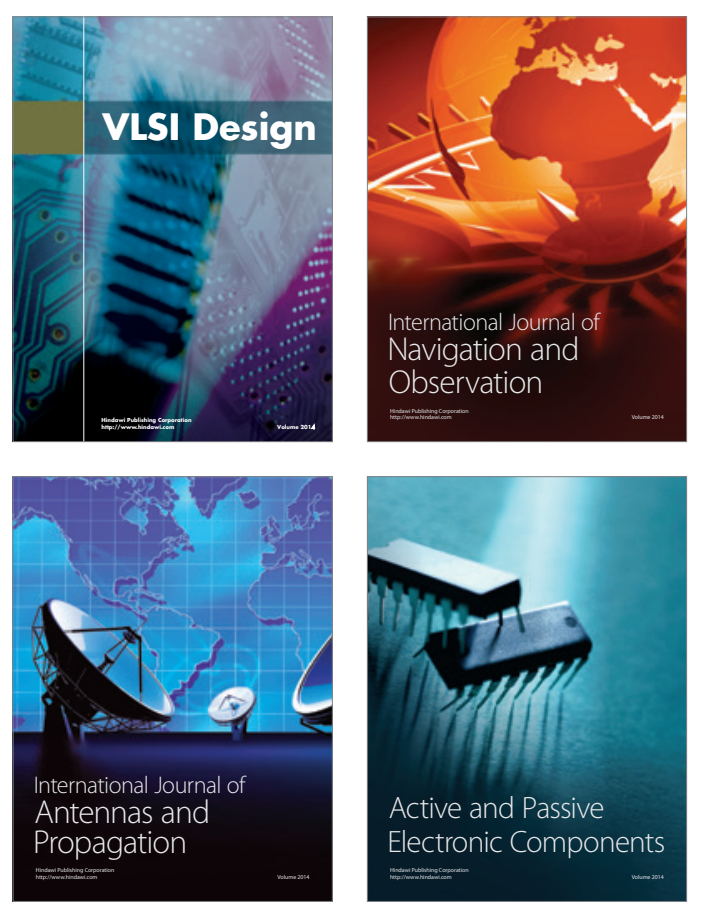
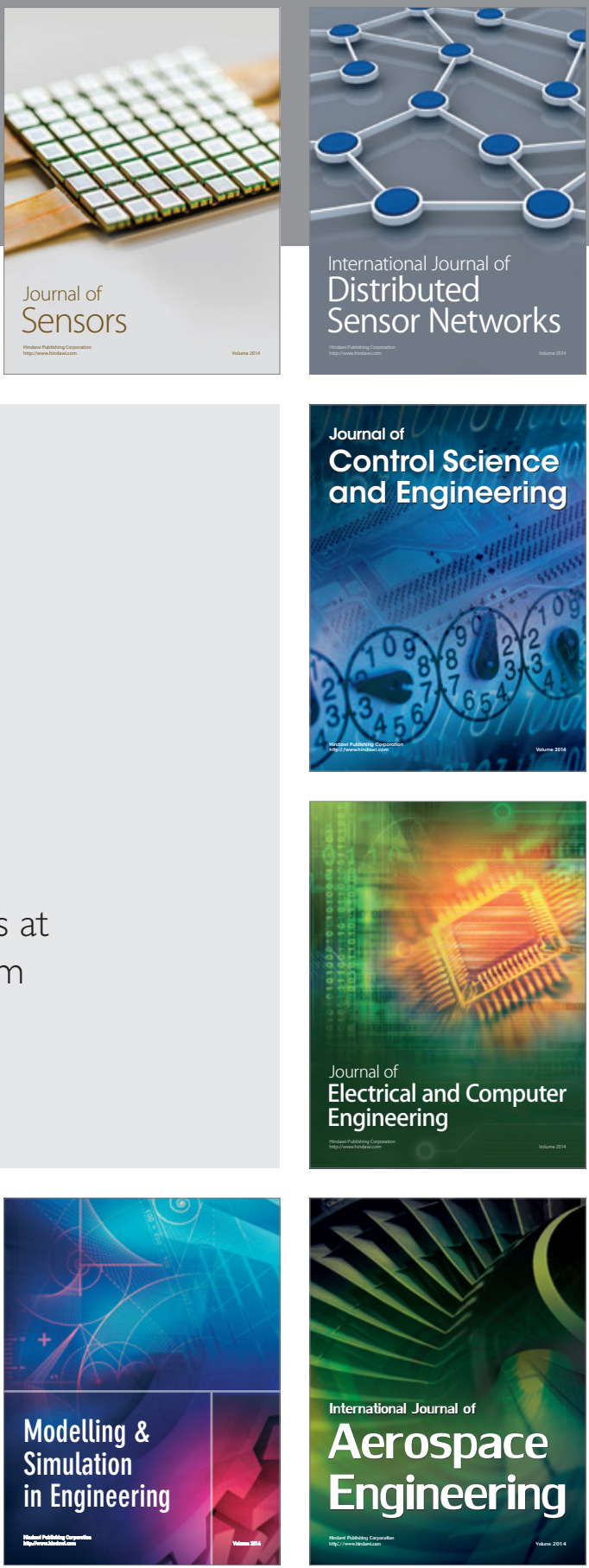

Journal of

Control Science

and Engineering
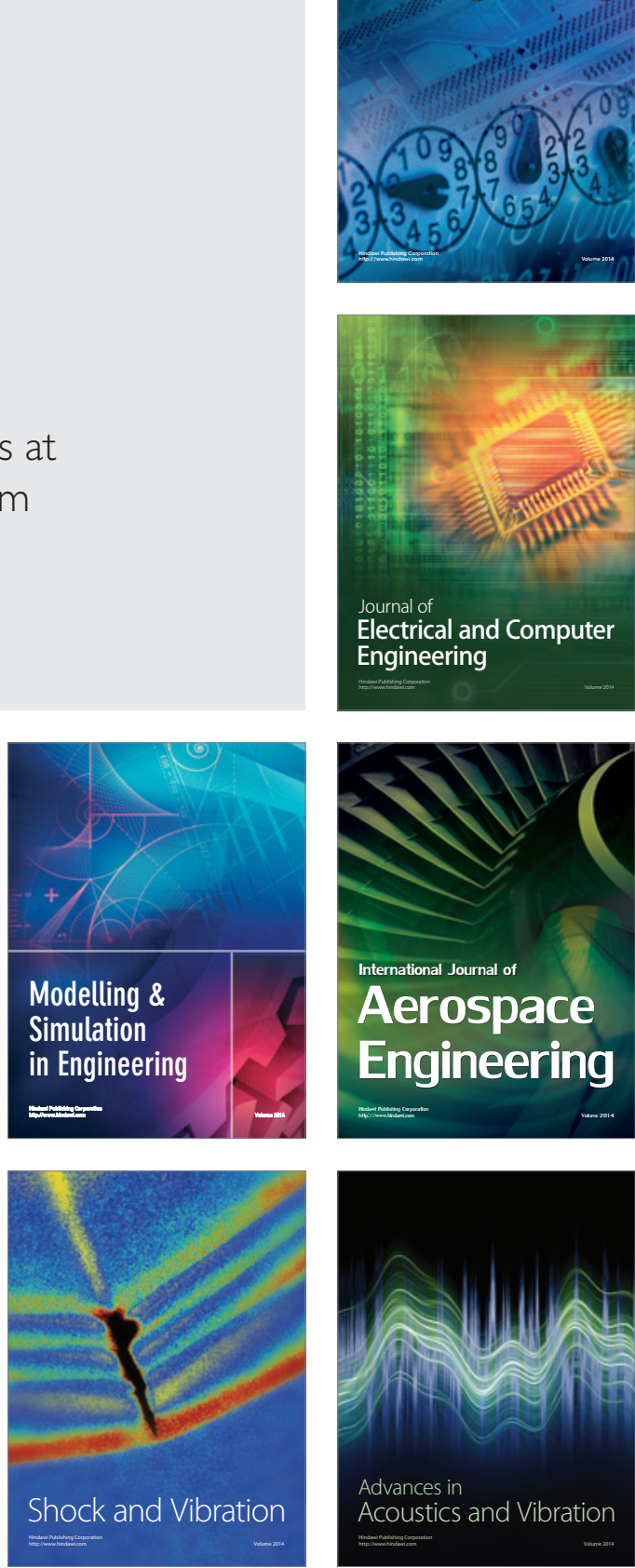